\title{
Role of Triplet States in the Photodynamics of Aniline
}

\author{
Aleksandr O. Lykhin ${ }^{1}$, Donald G. Truhlar, ${ }^{2 *}$ and Laura Gagliardi ${ }^{*}$ \\ ${ }^{1}$ Department of Chemistry, Pritzker School of Molecular Engineering, The James Franck Institute and \\ Chicago Center for Theoretical Chemistry, The University of Chicago, Chicago, IL 60637, United States \\ ${ }^{2}$ Department of Chemistry, Chemical Theory Center, and Minnesota Supercomputing Institute, University \\ of Minnesota, Minneapolis, MN 55455, United States \\ E-mail:1ykhin@uchicago.edu \\ E-mail: truhlar@umn.edu \\ E-mail: 1gagliardi@uchicago.edu
}

\begin{abstract}
The dynamics of excited heteroaromatic molecules is a key to understanding the photoprotective properties of many biologically relevant chromophores that dissipate their excitation energy nonreactively and thereby prevent the detrimental effects of ultraviolet radiation. Despite their structural variability, most heteroaromatic compounds share a common feature of a repulsive ${ }^{1} \pi \sigma^{*}$ potential energy surface. This surface can lead to photoproducts, and it can also facilitate the population transfer back to the ground electronic state by means of a ${ }^{1} \pi \sigma^{*} / \mathrm{S}_{0}$ conical intersection. Here, we explore a hidden relaxation route involving the triplet electronic state of aniline, which has recently been discovered by means of time-selected photofragment translational spectroscopy [J. Chem. Phys. 2019, 151, 141101]. By using the recently available analytical gradients for multiconfiguration pair-density functional theory, it is now possible to locate the minimum energy crossing points between states of different spin and therefore compute the intersystem crossing rates with a multireference method, rather than with the less reliable singlereference methods. Using such calculations, we demonstrate that the population loss of aniline in the $\mathrm{T}_{1}\left({ }^{3} \pi \pi^{*}\right)$ state is dominated by $\mathrm{C}_{6} \mathrm{H}_{5} \mathrm{NH}_{2} \rightarrow \mathrm{C}_{6} \mathrm{H}_{5} \mathrm{NH} \cdot+\mathrm{H} \cdot$ dissociation, and we explain the long nonradiative lifetimes of the $\mathrm{T}_{1}\left({ }^{3} \pi \pi^{*}\right)$ state at the excitation wavelengths of 294-264 nm.
\end{abstract}




\section{INTRODUCTION}

The dynamics of excited electronic states is of central importance in many branches of chemistry, and understanding how structural transformations control the dynamics in photoactivated molecules is one of the most exciting challenges in photochemistry, ${ }^{1,2}$ photovoltaics, ${ }^{3,4}$ bioimaging, ${ }^{5}$ photoredox catalysis, ${ }^{6,7}$ and light emitting technologies. ${ }^{8,9}$ Thanks to ultrashort laser pulses, it is now possible to experimentally probe not only the slow and moderatespeed dynamics but also the ultrafast processes taking place on the femtosecond or even attosecond time scales. ${ }^{10-12}$ The knowledge of the photodynamics mechanisms can then be used to tune the properties of excited states and design new materials, for example, more efficient photosensitizers. ${ }^{13,14}$ Furthermore, interpretation of experimental data can be improved by insights from computational modeling methods to make assignments and disentangle intricate spectroscopic features. $^{15}$

Excited states are difficult targets for computational study because they usually involve significant mixing of electron configurations, and this mixing becomes most severe in regions where two electronic states become degenerate or nearly degenerate, ${ }^{16,17}$ which are the most important regions for interstate population transfer. ${ }^{18}$ Single-reference methods are generally inadequate in the vicinity of such intersections, and they often even fail to provide the correct topography of the potential energy landscape. ${ }^{19-21}$ Even if two crossing states have different spins and therefore do not mix in the nonrelativistic approximation, the use of single-reference methods can still be questionable as one or both of the two states can be inherently multiconfigurational. Thus, it is advisable to use multireference methods when searching for the conical intersections (CI) or intersystem crossing (ISC) seams.

Among multireference methods, a widely preferred choice to treat excited states is second-order complete-active-space perturbation theory ${ }^{22,23}$ (CASPT2) or its extended multistate version $^{24,25}$ (XMS-CASPT2). These methods often have good accuracy, and in some programs there are analytic gradients, which are helpful in optimization and dynamics. ${ }^{26,27}$ However, multireference perturbation theories are usually limited to molecules of moderate size because of the post-SCF iterative perturbation step, which requires large memory due to the need for higher-order density matrices. A more cost-effective approach to deal with multireference systems is multiconfiguration 
pair-density functional theory (MC-PDFT). ${ }^{28,29} \mathrm{In}$ MC-PDFT, the post-SCF step is noniterative and requires only the kinetic energy, electron density and its gradient, and on-top pair density (and optionally the gradient of the on-top pair density) from a multiconfigurational wave function (usually a multiconfigurational self-consistent field (MCSCF) method) without needing higherorder density matrices. While the accuracy of MC-PDFT is comparable to CASPT2, its computational cost is largely reduced to the order of the MCSCF step. ${ }^{30,31}$ MC-PDFT also overcomes some shortcomings of Kohn-Sham density functional theory (KS theory), for example, unphysical spin mixing in open-shell electronic states, ${ }^{32,33}$ or strong dependence of the predicted singlet-triplet gaps upon the amount of Hartree-Fock exchange in the exchange-correlation functional. ${ }^{34,35}$ Another advantage of MC-PDFT compared to KS theory is the elimination of selfinteraction error. ${ }^{36}$ MC-PDFT has shown good accuracy for the correct ordering of electronic states, accurate electronic energy gaps, and good estimates of barrier heights. ${ }^{30,37-39}$ The recent implementation of the state-specific ${ }^{40}$ and state-averaged ${ }^{41}$ analytical gradients makes MC-PDFT an appealing alternative to KS theory and CASPT2 for modeling ISC phenomena or predicting properties of excited electronic states.

Here, we use MC-PDFT to elucidate the participation of triplet states in the radiationless relaxation of aniline, which is important for understanding the photostability of aromatic biomolecules containing N-H groups. ${ }^{42-45}$ Since the aniline chromophore also occurs in paraaminobenzoic acid and methyl anthranilate, which are commonly used to prevent skin damage from excessive ultraviolet radiation, this relaxation dynamics is also of interest for developing and understanding sunscreen photoprotectors. ${ }^{46}$ For a long time, it was believed that the single-photon dissociation of aniline triggered by ultraviolet light occurs exclusively via population transfer to the dissociative region of the ${ }^{1} \pi \sigma^{*}$ excited electronic state. ${ }^{47-50}$ However, experiments employing a new time-selected photofragment translational spectroscopy method revealed a previously unknown dissociation mechanism in the dark triplet state accessible via ISC from the $\mathrm{S}_{1}$ state optically populated at $5.00 \mathrm{eV}(248 \mathrm{~nm}){ }^{51}$ The question arises of the precise mechanism for this triplet participation, and here we use the new gradient capability of MC-PDFT to investigate aniline dynamics once the system reaches the $\mathrm{T}_{1}$ state. In particular, we calculate the rate constants for the 
nonreactive ISC process $\mathrm{T}_{1} \rightarrow \mathrm{S}_{0}$ and for the dissociative electronically adiabatic process $\mathrm{T}_{1} \rightarrow$ $\mathrm{C}_{6} \mathrm{H}_{5} \mathrm{NH} \cdot+\mathrm{H} \cdot$

\section{COMPUTATIONAL METHODS}

2.1. Electronic structure. MC-PDFT and extended multi-state PDFT ${ }^{52}$ (XMS-PDFT) calculations were performed in the OpenMolcas $^{53}$ (v19.11, tag 1553-g2c87dca-dirty) suite of programs, whereas the unrestricted KS theory and spin-orbit coupling calculations were carried out in the GAMESS ${ }^{54}$ (version June 30, $2020 \mathrm{R} 1$ ) software package.

The critical geometries were optimized with KS theory and MC-PDFT without imposing any symmetry constraints. In the case of MC-PDFT, the translated ${ }^{28}$ and fully-translated ${ }^{55}$ on-top density functionals, tPBE and ftPBE, were employed, and a CASSCF wave function ${ }^{56}$ was used as the SCF reference function. We considered three active spaces: (i) a small one $(6,6)$ with six electrons in three $\pi$ bonding orbitals and three $\pi^{*}$ antibonding orbitals, (ii) a medium one $(12,12)$ that included all orbitals of the small active space plus the nitrogen lone-pair orbitals $2 p_{z}(N)$ and $3 \mathrm{p}_{\mathrm{z}}(\mathrm{N})$, the bonding orbitals $\sigma(\mathrm{C}-\mathrm{N})$ and $\sigma(\mathrm{N}-\mathrm{H})$, and their antibonding counterparts $\sigma^{*}(\mathrm{C}-\mathrm{N})$ and $\sigma^{*}(\mathrm{~N}-\mathrm{H})$, and (iii) a large one $(14,14)$ obtained from $(12,12)$ active space by adding the second pair of $\sigma(\mathrm{N}-\mathrm{H})$ and $\sigma^{*}(\mathrm{~N}-\mathrm{H})$ orbitals. The electronic energies of the high-lying excited singlet and triplet states were found with XMS(3)-tPBE calculations, based on state-averaged CASSCF where energies were averaged over the three states with the equal weights, and where we used a threestate model space for the multi-state treatment. We used two density functionals for KS calculations: $\mathrm{PBE}^{57}$ and MN15. ${ }^{58}$ The def2-TZVP basis set ${ }^{59}$ was used throughout this work. We used the fine integration grid $(75,302)$ for PBE, MC-PDFT, and XMS-PDFT and the ultrafine grid $(99,590)$ for MN15.

The spin-orbit coupling at the triplet-singlet crossing points was computed using the full two-electron Breit-Pauli spin-orbit Hamiltonian coupled with the complete active space configuration interaction (CASCI) method. ${ }^{60}$ The initial molecular orbitals were prepared using the state-averaged CASSCF method by averaging over the $T_{1}$ and $S_{0}$ crossing states having equal weights in the spin-orbit coupling calculation performed with GAMESS. 
2.2. Dynamics. The rate constants were computed using microcanonical nonadiabatic transition state theory ${ }^{61}\left(\mu \mathrm{NA}\right.$-TST) for the $\mathrm{T}_{1} \rightarrow \mathrm{S}_{0}$ ISC process and using Rice-RamspergerKassel-Marcus (RRKM) theory ${ }^{62-65}$ with the zero-curvature tunneling (ZCT) approximation ${ }^{66,67}$ for the electronically adiabatic dissociation in the triplet state. These dynamics calculations were carried out in part using the master equation program MESMER ${ }^{68}$ Note that RRKM theory is microcanonical transition state theory ${ }^{69,70}$ applied to a unimolecular reaction, and $\mu$ NA-TST is the extension of this theory to electronically nonadiabatic processes. ${ }^{71-77}$

The ISC rate constant, $k_{\mathrm{ISC}}$, and unimolecular dissociation rate constant, $k_{\mathrm{Dis}}$, were computed using

$$
\begin{aligned}
& k_{I S C}(E)=\frac{f}{h \rho_{R}(E)} \int_{0}^{E} \rho_{X}(E-\varepsilon) P_{I S C}(\varepsilon) d \varepsilon, \\
& k_{D i s}(E)=\frac{f}{h \rho_{R}(E)} \int_{0}^{E} \rho_{T S}(E-\varepsilon) P_{T r}(\varepsilon) d \varepsilon,
\end{aligned}
$$

where $E$ is the rovibrational energy in the molecule (taken from experiment), $h$ is Planck's constant, $f$ is the degeneracy of the reaction path, $\varepsilon$ is the energy in the reaction coordinate, $P_{I S C}$ is the probability of transition between two states of different spin, $P_{T r}$ is the barrier transmission probability, $\rho_{R}$ is the density of rovibrational of states of the reactant, $\rho_{X}$ is the rovibrational density of states in the transition-state theory dividing surface through the minimum energy crossing point (MECP) of $\mathrm{T}_{1}$ and $\mathrm{S}_{0}$, and $\rho_{T S}$ is the rovibrational density of states in the transition-state theory dividing surface through the saddle point for electronically adiabatic dissociation in the $\mathrm{T}_{1}$ state. The energy $E$ is partitioned into the reaction coordinate $(\varepsilon)$ and the remaining degrees of freedom $(E-\varepsilon)$. The reaction-coordinate motion is normal to the crossing seam in Eq. 1 and the transition state dividing surface in Eq. 2. The zero of energy $E$ is consistently taken as the zero-point energy level of the $\mathrm{T}_{1}$ state in all equations and discussion throughout this article.

To evaluate the probability $P_{I S C}$ of transition between $\mathrm{T}_{1}$ and $\mathrm{S}_{0}$ when a trajectory passes through their crossing seam, we used the weak coupling formula: ${ }^{78-81}$

$$
P_{I S C}(\varepsilon)=\alpha \operatorname{Ai}^{2}(\beta),
$$




$$
\begin{gathered}
\alpha=H_{S O C}^{2}\left(\frac{32 \mu \pi^{5}}{h^{2} \sqrt{\left|\mathbf{g}_{1}\right|\left|\mathbf{g}_{2}\right|}\left|\mathbf{g}_{1}-\mathbf{g}_{2}\right|}\right)^{2 / 3}, \\
\beta(\varepsilon)=-\left(\varepsilon-E_{X}-Z P E_{X}\right)\left(\frac{\pi \sqrt{8 \mu}\left|\mathbf{g}_{1}-\mathbf{g}_{2}\right|}{h\left|\mathbf{g}_{1}\right|\left|\mathbf{g}_{2}\right|}\right)^{2 / 3},
\end{gathered}
$$

where $\operatorname{Ai}(\beta)$ is the Airy function, $H_{S O C}$ is the spin-orbit coupling constant (SOC) at the crossing seam (assumed to be locally constant), $\mu$ is the reduced mass of the reaction coordinate (see Appendix), $\mathbf{g}_{1}$ and $\mathbf{g}_{2}$ are the gradients of $\mathrm{T}_{1}$ and $\mathrm{S}_{0}$ crossing surfaces, $E_{X}$ is the energy of the minimum energy crossing point with respect to the zero-point energy level of the $\mathrm{T}_{1}$ reactant, and $Z P E_{X}$ is the zero-point energy at the MECP.

Note that $\mathbf{g}_{1}-\mathbf{g}_{2}$ is normal to the singlet-triplet crossing seam at the MECP. To find the direction normal to the crossing seam, we compute an effective mass-weighted Hessian that arises from the second-order Taylor expansion for the energy of both states in the vicinity of the MECP for a displacement along the crossing seam. This Hessian has only one imaginary frequency at the MECP with the corresponding eigenvector being orthogonal to the crossing seam and collinear with the reaction coordinate; details are given in the Appendix.

When calculating dissociation rates, the probability of transmission $P_{T r}$ through the barrier was estimated using the zero-curvature tunneling approximation, implemented semiclassically ${ }^{82}$ as

$$
\begin{gathered}
P_{T r}(\varepsilon)=\frac{1}{1+\exp (\theta(\varepsilon))} \\
\theta(\varepsilon)=\frac{4 \pi}{h} \int_{s<}^{s>} \sqrt{2 \mu\left(V_{\mathrm{MEP}}(s)+Z P E(s)-\varepsilon\right)} d s, \varepsilon<V_{0} \\
V_{0} \equiv V_{\mathrm{MEP}}(s=0)+Z P E(s=0)
\end{gathered}
$$

where the reaction coordinate is calculated as the arc length along the minimum energy path (MEP) in mass-scaled coordinates ${ }^{83}$ with the reduced mass $\mu$ set to 1 amu and with $s=0$ at the saddle point, $s_{<}$and $s_{>}$are the classical turning points, $V_{\text {MEP }}(s)$ is the potential energy along the MEP relative to the zero-point energy level of the $\mathrm{T}_{1}$ reactant, and $Z P E(s)$ is the zero-point energy in modes transverse to the reaction coordinate. In eq (7), we approximated $Z P E(s)$ as $Z P E(s=0)$. 
The rovibrational densities of states were calculated using the quantum asymmetric top and harmonic oscillator models with an integration step size of $10 \mathrm{~cm}^{-1}$. When calculating dissociation rates with $\mathrm{ftPBE}(14,14)$, we used vibrational frequencies and minimum energy path geometries obtained with $\operatorname{tPBE}(14,14)$. Subsequently, ftPBE $(14,14)$ single-point calculations were carried out along the dissociation path, while the transition state and the equilibrium geometry were reoptimized explicitly with ftPBE(14,14). The MC-PDFT Hessians were computed seminumerically by evaluating finite differences between the components of the analytical gradients in a homemade Python script. We confirmed that the Hessians yielded no imaginary frequencies at the minima and only one at the transition states.

\section{RESULTS AND DISCUSSION}

The $\mathrm{T}_{1}$ lifetimes of collision-free aniline were originally measured by Knee and Johnson using the pump-probe technique coupled with time-of-flight mass spectrometry. ${ }^{84}$ In that experiment, the first excited triplet state $\mathrm{T}_{1}$ was populated through the ISC from the higher-lying $\mathrm{S}_{1}$ vibronic states produced by ultraviolet irradiation of the jet-cooled aniline. Overall, eleven $\mathrm{S}_{1}$ vibronic states were prepared at excitation energies from $4.22 \mathrm{eV}(294 \mathrm{~nm})$ to $4.70 \mathrm{eV}(264 \mathrm{~nm})$ as summarized in Table 1. These energies are generally too low to populate the close-lying $\mathrm{S}_{2}\left(\pi \sigma^{*}\right)$ excited state with the origin at $4.60 \mathrm{eV} .{ }^{85}$ In the case of the high-lying vibronic state at $4.69 \mathrm{eV}$, the direct $\mathrm{S}_{0} \rightarrow \mathrm{S}_{2}\left(\pi \sigma^{*}\right)$ excitation is also unlikely because of the four-fold decrease of the oscillator strength compared to the $\mathrm{S}_{0} \rightarrow \mathrm{S}_{1}\left(\pi \pi^{*}\right)$ transition. ${ }^{50,86,87}$

Once formed, $\mathrm{S}_{1}$ vibronic states decay rapidly via multiple relaxation mechanisms including the $\mathrm{S}_{1} \rightarrow \mathrm{S}_{0}$ fluorescence, $\mathrm{S}_{1} \rightarrow \mathrm{S}_{0}$ internal conversion (IC), and ISC to the triplet manifold as schematically illustrated in the upper panel of Figure 1. In addition to these three channels, aniline can also undergo dissociation into anilino radical $\left(\mathrm{C}_{6} \mathrm{H}_{5} \mathrm{NH} \cdot\right)$ and atomic hydrogen. However, at the low excitation energies, such dissociation is expected to be inefficient because of the barrier formed by the bound $S_{1}\left({ }^{1} \pi \pi^{*}\right)$ and quasi-bound $S_{2}\left({ }^{1} \pi \sigma^{*}\right)$ excited states crossing one another along the N-H stretching coordinate. ${ }^{50}$ Using extended multiconfiguration quasi-degenerate perturbation theory (XMCQDPT2), the barrier height for dissociation of aniline in the lowest excited singlet state $\mathrm{S}_{1}$ was found to be $0.41 \mathrm{eV}$ at the geometries optimized by SA(3)-CASSCF(10,9)/6-311++G**. ${ }^{88}$ This 
barrier height suggests that all $\mathrm{S}_{1}$ vibronic states probed by Knee and Johnson, except the $12_{0}^{4}$ state with four vibrational quanta in the in-plane ring deformation mode, would have to tunnel through the barrier in order to end up in the $\pi \sigma^{*}$ repulsive region of the $S_{1}$ PES. In accord with the computational arguments, no evidence of ultrafast N-H fission across the 294-270 nm excitation range were found in time-resolved velocity map imaging experiment. ${ }^{50}$ Thus, the nanosecond decay of the $S_{1}$ state $(<10 \mathrm{~ns})$ at the low excitation energies is dominated by fluorescence and nonradiative relaxation either to the ground state or to the excited triplet states ${ }^{84,89}$ shown in the upper panel of Figure 1.

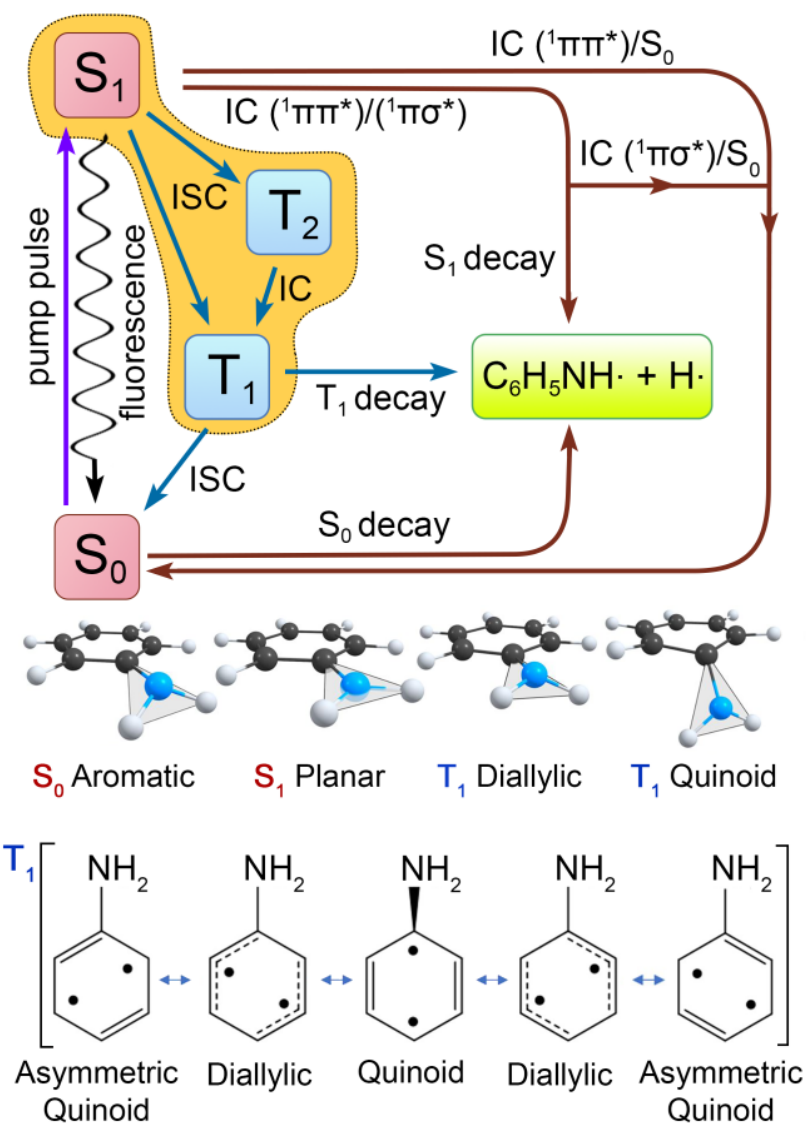

Figure 1. Upper panel: Relaxation pathways from the optically-populated $S_{1}\left(\pi \pi^{*}\right)$ state of aniline to the ground state $\mathrm{S}_{0}$ and anilino radical, $\mathrm{C}_{6} \mathrm{H}_{5} \mathrm{NH} \cdot$, and critical geometries of aniline. The states highlighted by orange background could be ionized in the pump-probe experiment by Knee and Johnson. Lower panel: Resonance structures of $\mathrm{T}_{1}$ aniline with the high spin density sites denoted by black dots. 
Although it is not clear which of the two low-lying triplets is populated through the ISC from the $S_{1}$ state, most of the population is expected to be accumulated in the $T_{1}$ state. This assumption is supported by the fact that in the vicinity of $S_{0} \rightarrow T_{1}$ Franck-Condon region the $T_{2}$ and $\mathrm{T}_{1}$ states approach one another and become degenerate at about $0.61 \mathrm{eV}$ above the $\mathrm{T}_{1}$ equilibrium as deduced from the single-point XMS-tPBE(14,14) calculations carried out at the geometries optimized with SA-tPBE $(14,14)$ (see Figure S4 in SI). This energy provides an upper bound for the $\mathrm{T}_{2}\left({ }^{3} \pi \pi^{*}\right) / \mathrm{T}_{1}\left({ }^{3} \pi \pi^{*}\right)$ conical intersection mediating population transfer from the $\mathrm{T}_{2}$ state to the lowest triplet state $T_{1}$. The presence of the low-lying $T_{1}$ excited state at the energy lower than the $S_{1}$ origin was also confirmed experimentally by measuring the phosphorescence spectrum of aniline in a rigid glass solution of EPA (a mixture of diethyl ether, isopentane and ethanol in 5:5:2 ratio). In particular, the band centered at $\approx 3.32 \mathrm{eV}$ was attributed to the $0-0$ emission from $\mathrm{T}_{1}$ to $\mathrm{S}_{0}$, whereas no signature of the high-lying triplets was observed. ${ }^{90,91}$

In order to probe the $\mathrm{T}_{1}$ decay Knee and Johnson used ultraviolet radiation to reach the ionization potential ${ }^{92}$ of aniline at $7.72 \mathrm{eV}$ and produce ions to be captured by the time-of-flight mass spectrometer. Both $T_{1}$ and $S_{1}$ states were ionized by the probe pulses keeping intact the hot $S_{0}$ aniline molecules. Because $S_{1}$ decayed faster than $T_{1}$, they fit the overall population in two states to a single exponential, except for the cases when the $S_{1}$ contribution was noticeable and where two exponentials were used. Due to the lack of phosphorescence, the experimentally measured microsecond lifetimes were attributed to the nonradiative decay of $T_{1}$. Using the gap between $T_{1}$ origin in EPA and optically populated $S_{1}$ vibronic states in the gas phase, Knee and Johnson found internal rovibrational energies in $\mathrm{T}_{1}$ aniline to range from 0.90 to $1.37 \mathrm{eV}$ (Table 1). However, these energies are likely to deviate from those in the jet-cooled aniline due to the perturbation caused by EPA on the $\mathrm{T}_{1}$ origin. Indeed, more recent spectroscopic measurements show that the electronic excitations in aniline are quite sensitive to the host environment. For example, a well-defined $\mathrm{S}_{1}$ origin at $4.22 \mathrm{eV}$ in the gas phase ${ }^{84,93}$ becomes red-shifted by $0.11 \mathrm{eV}$ in cyclohexane and by 0.19 $\mathrm{eV}$ in benzene at $77 \mathrm{~K} .{ }^{93} \mathrm{In}$ the argon matrix, on the other hand, the host-guest interaction is weak and the $\mathrm{S}_{1}$ origin at $4.21 \mathrm{eV}$ is redshifted by only $0.01 \mathrm{eV}$ at $10 \mathrm{~K} .{ }^{94} \mathrm{We}$ believe that an even smaller redshift can be expected for the $T_{1}$ origin because it is less sensitive to the perturbation than the $S_{1}$ state. In fact, taking argon matrix as a reference, it can be seen that $S_{1}$ is red-shifted in the benzene 
and cyclohexane glasses by 0.18 and $0.10 \mathrm{eV}$, while $\mathrm{T}_{1}$ is redshifted by only 0.05 and $0.04 \mathrm{eV}$, respectively. ${ }^{93,94}$ In a similar way, the low-lying electronic states in structurally relevant benzene are less perturbed by matrix than the high-lying states. The three absorption bands in benzene gas ${ }^{1} \mathrm{~A}_{1 \mathrm{~g}} \rightarrow{ }^{1} \mathrm{E}_{1 \mathrm{u}}(6.87 \mathrm{eV}),{ }^{1} \mathrm{~A}_{1 \mathrm{~g}} \rightarrow{ }^{1} \mathrm{~B}_{1 \mathrm{u}}(6.10 \mathrm{eV})$, and ${ }^{1} \mathrm{~A}_{1 \mathrm{~g}} \rightarrow{ }^{1} \mathrm{~B}_{2 \mathrm{u}}(4.79 \mathrm{eV})$ become redshifted in solid argon by $0.15,0.09$, and $0.01 \mathrm{eV}$, respectively. ${ }^{95}$ Thus, the gap between $\mathrm{T}_{1}$ and $\mathrm{S}_{1}$ states of $0.76 \mathrm{eV}$ in the argon matrix provides a lower bound to the gap in the jet-cooled aniline when two states are systematically shifted to the lower energies. The upper bound of $0.77 \mathrm{eV}$ corresponds to the case where, in contrast to the redshifted $S_{1}$, the $T_{1}$ origin is not perturbed by the argon matrix. Using the $\mathrm{T}_{1} / \mathrm{S}_{1}$ gap in argon, $E_{\mathrm{S}_{1}-\mathrm{T}_{1}}$, and $\mathrm{S}_{1}$ rovibrational energies measured by Knee and Johnson for the jet-cooled aniline, $E_{\mathrm{S}_{1}}$, we calculated rovibrational energies in the $\mathrm{T}_{1}$ state as

$$
E_{\mathrm{T}_{1}}=E_{\mathrm{S}_{1}}+E_{\mathrm{S}_{1}-\mathrm{T}_{1}} \text {. }
$$

These energies are reported in the "Corrected" column of Table 1. Note that the assignment of vibronic levels follows Castella-Ventura and $\mathrm{Kassab}^{96}$ rather than that of Knee and Johnson.

Table 1. Excitation energies of $S_{1}$ vibronic states in jet-cooled aniline, energy above $\mathrm{S}_{1}$ origin $\left(E_{\mathrm{S}_{1}}\right)$, energy above $\mathrm{T}_{1}\left(E_{\mathrm{T}_{1}}\right)$, and $\mathrm{T}_{1}$ nonradiative lifetimes.

\begin{tabular}{|c|c|c|c|c|c|c|c|}
\hline \multicolumn{3}{|c|}{$\mathrm{S}_{1}$ vibronic transitions in jet ${ }^{84}$} & \multicolumn{2}{|c|}{$E_{\mathrm{S}_{1}}(\mathrm{eV})$} & \multicolumn{2}{|c|}{$E_{\mathrm{T}_{1}}(\mathrm{eV})^{b}$} & \multirow{2}{*}{$\mathrm{T}_{1}$ lifetimes $(\mathrm{ms})^{84}$} \\
\hline Assignment $^{a}$ & $\lambda(\mathrm{nm})$ & $\Delta E(\mathrm{eV})$ & in jet ${ }^{84}$ & in $\mathrm{Ar}^{94}$ & $\left(\right.$ ref $\left.^{84}\right)$ & Corrected & \\
\hline $0_{0}^{0}$ & 293.8 & 4.22 & 0.00 & 0.00 & 0.90 & 0.76 & 5.65 \\
\hline $6 a_{0}^{1}$ & 289.7 & 4.28 & 0.06 & 0.06 & 0.96 & 0.82 & 4.20 \\
\hline $12_{0}^{1}$ & 285.9 & 4.34 & 0.12 & 0.12 & 1.01 & 0.87 & 3.44 \\
\hline $20 a_{0}^{1}$ & 283.0 & 4.38 & 0.16 & 0.16 & 1.06 & 0.92 & 2.61 \\
\hline $1_{0}^{2}$ & 280.7 & 4.42 & 0.20 & 0.20 & 1.09 & 0.95 & 2.09 \\
\hline $\mathrm{I}_{0}^{2} 12_{0}^{1}$ & 279.8 & 4.43 & 0.21 & & 1.11 & 0.969 & 1.90 \\
\hline $1_{0}^{1} 12_{0}^{1}$ & 279.5 & 4.44 & 0.22 & 0.22 & 1.11 & 0.974 & 1.83 \\
\hline $12_{0}^{2}$ & 278.3 & 4.46 & 0.24 & & 1.13 & 0.99 & 1.63 \\
\hline $12_{0}^{1} 20 a_{0}^{1}$ & 275.6 & 4.50 & 0.28 & 0.28 & 1.18 & 1.04 & 1.19 \\
\hline $12_{0}^{3}$ & 271.1 & 4.57 & 0.35 & & 1.25 & 1.11 & 0.62 \\
\hline $12_{0}^{4}$ & 264.3 & 4.69 & 0.47 & & 1.37 & 1.23 & 0.167 \\
\hline
\end{tabular}

${ }^{a}$ The assignment of vibronic levels follows that of Castellá-Ventura and Kassab. ${ }^{96}$

${ }^{b}$ This is the energy scale used in the rest of this article. 
As shown in Table 1, the $\mathrm{T}_{1}$ lifetimes measured in the pump-probe experiment drop down significantly with the increase of the internal energy in the $\mathrm{T}_{1}$ state approaching $168 \mathrm{~ns}$ at the excitation energy of $4.69 \mathrm{eV}$. Taking the inverse of these lifetimes the corresponding unimolecular rate constants are as small as $10^{5}-10^{6} \mathrm{~s}^{-1}$, which can be explained, for example, by the dissociation of aniline in the $T_{1}$ state or the inefficient $T_{1} \rightarrow S_{0}$ ISC that is typical for organic molecules. Unfortunately, the long experimental lifetimes of $\mathrm{T}_{1}$ state preclude the use of nonadiabatic molecular dynamics ${ }^{97-99}$ to get insight into kinetics measured by Knee and Johnson and answer the question of whether the relaxation dynamics of aniline involves dissociation in the triplet state upon ultraviolet irradiation. Therefore, we exploit statistical theories such as $\mu$ NA-TST and RRKM with input from electronic structure calculations.

In the ground singlet state, aniline is an aromatic molecule with nearly equal $\mathrm{C}-\mathrm{C}$ bond lengths and the nitrogen lone-pair being almost in-line with the $\pi$-system of the ring. The excitation to the $T_{1}$ state is mainly associated with $\pi \rightarrow \pi^{*}$ transitions which disturb the conjugation between the aromatic system and nitrogen. The state-specific $\operatorname{tPBE}(14,14)$ calculations confirm two minima on the $\mathrm{T}_{1}$ PES corresponding to the diallylic and quinoid configurations (Figure 1, lower panel). The ZPE-inclusive energies reported in Table 2 show that the diallylic conformer is slightly more favorable than the symmetric quinoid, although the difference is quite small $(0.03 \mathrm{eV})$. In the diallylic configuration, the nitrogen atom is slightly tilted by $3^{\circ}$ from the plane of the phenyl ring, while in the symmetric quinoid it is out-of-plane by almost $40^{\circ}$. Due to the nitrogen lone pair, the amino group adopts a pyramidal shape in both conformers as in ammonia. The PBE functional does not predict a correct diallylic conformer, but rather yields an asymmetric quinoid with one of the two hydrogens in the ortho position distorted by $16.1^{\circ}$ from the plane of the phenyl ring and two C-C allylic bonds of 1.38 and $1.42 \AA$ next to the amino group, which are expected to be formally equivalent (see SI, Figure S5). This is in contrast to the out-of-plane angle of $3.0^{\circ}$ and corresponding bonds of 1.38 and $1.39 \AA$ A found with the tPBE and ftPBE on-top functionals. The incorrect geometry predicted by PBE can be explained by overestimation of $\mathrm{sp}^{3}$-hybridization at the ortho carbon. This shows that the triplet aniline is a challenging system for single-reference methods because the wave function in both diallylic and symmetric quinoid conformers has a substantial multiconfigurational character with the leading determinants having weights of 0.81 and 0.84 , respectively. The 
multiconfigurational character of triplet aniline is also manifested by the partially occupied natural orbitals. For example, in the diallylic conformer the occupation numbers are 1.92 and 1.88 for the doubly occupied $\pi$-orbitals, 1.05 and 0.95 for the singly occupied orbitals, and 0.08 and 0.12 for the unoccupied $\pi^{*}$-orbitals as illustrated in Figure 2. Unlike PBE, the MN15 functional predicts hydrogen distortion angle of $4.1^{\circ}$ and $\mathrm{C}-\mathrm{C}$ allylic bond lengths of 1.37 and $1.40 \AA$, which are much closer to the values found with $\operatorname{PBBE}(14,14)$ and $\operatorname{ftPBE}(14,14)$. Such behavior is consistent with the fact that MN15 functional is more accurate than PBE for multireference systems. ${ }^{58}$ The $0-0$ gap between the $T_{1}$ and $S_{0}$ states predicted by tPBE and ftPBE is larger than the experimental value of $3.45 \mathrm{eV}$ in the argon matrix by almost 0.19 and $0.12 \mathrm{eV}$, respectively. This is consistent with the destabilization of the high-spin states that has been previously reported for the tPBE functional. ${ }^{31}$

Table 2. Energies of the key structures $(\mathrm{eV})$ with respect to the ZPE level of the $\mathrm{T}_{1}$ diallylic minimum and the bond lengths $(\AA)$ at the transition states on the $\mathrm{T}_{1} \mathrm{PES}$.

\begin{tabular}{lccccc}
\hline \hline & MN15 & PBE & tPBE $(6,6)$ & tPBE $(14,14)$ & $\mathrm{ftPBE}(14,14)$ \\
\hline $\mathrm{T}_{1}$ (diallylic) & 0 & 0 & 0 & 0 & 0 \\
$\mathrm{~T}_{1}$ (quinoid) & 0.05 & 0.03 & 0.03 & 0.03 & 0.03 \\
$\mathrm{~S}_{0}$ (aromatic) & -3.46 & -3.27 & -3.70 & -3.64 & -3.57 \\
$\mathrm{~T}_{1}-\mathrm{S}_{0}$ MECP A & 0.28 & 0.51 & 0.40 & 0.41 & $0.40^{a}$ \\
$\mathrm{~T}_{1}-\mathrm{S}_{0}$ MECP B & 0.28 & 0.51 & 0.40 & 0.42 & $0.40^{a}$ \\
$\mathrm{~T}_{1}-\mathrm{S}_{0}$ MECP C & 0.99 & 0.94 & 0.96 & 0.95 & $0.99^{a}$ \\
$\mathrm{~T}_{1}-\mathrm{S}_{0}$ MECP D & 0.26 & 0.50 & 0.43 & 0.45 & $0.42^{a}$ \\
$\mathrm{~T}_{1} \mathrm{TS}(\mathrm{C}-\mathrm{N})$ & 1.50 & 1.30 & & 1.29 & 1.30 \\
$\mathrm{~T}_{1} \mathrm{TS}$ (N-H) & 1.04 & 0.68 & & 0.76 & 0.80 \\
& & & & & \\
$\mathrm{C}-\mathrm{N}$ bond length & 2.017 & 2.253 & - & 2.023 & 2.023 \\
$\mathrm{~N}-\mathrm{H}$ bond length & 1.445 & 1.556 & - & 1.492 & 1.490 \\
\hline \hline
\end{tabular}

${ }^{a}$ The MECP entries in the final column are calculated by $\operatorname{ftPBE}(14,14) / / \operatorname{PBE}(14,14)$ with single-point energies averaged over $S_{0}$ and $T_{1}$ states and vibrational frequencies calculated from $\operatorname{PBE}(14,14)$. 


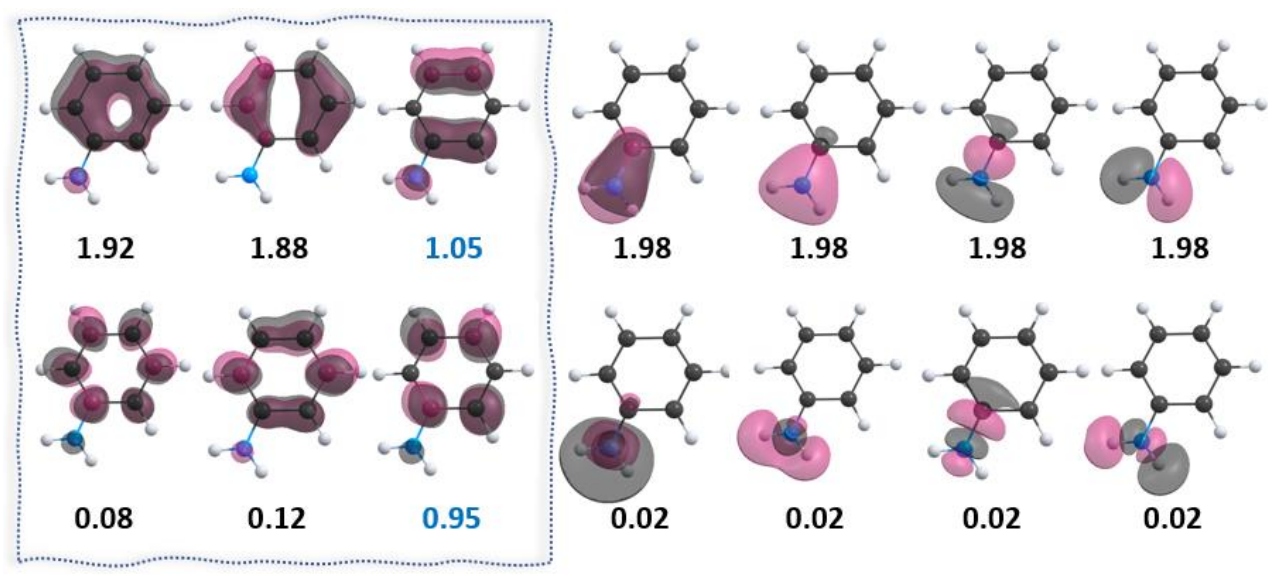

Figure 2. Natural orbitals and their occupation numbers at the $\operatorname{PBE}(14,14) \mathrm{T}_{1}$ diallylic minimum. The small active space is outlined in the left-hand side.

In order to calculate $\mathrm{T}_{1} \rightarrow \mathrm{S}_{0}$ ISC rate constants using $\mu \mathrm{NA}-\mathrm{TST}$, we first searched for minimum energy crossing points (MECPs), which are local minima on the multidimensional crossing seam between the $T_{1}$ and $S_{0}$ PESs. Direct interpolation between $T_{1}$ and $S_{0}$ minima found no sign of the crossing states, but we found four such MECPs at energies up to $0.12 \mathrm{eV}$ above the $\mathrm{T}_{1}$ origin by sampling the vibrational space in the vicinity of the diallylic and symmetric quinoid minima; they are listed in Table 2. As shown in Figure 3, all MECPs arise from deformational vibrations breaking of the conjugation within the phenyl ring. (This is the first time that MECPs have been studied with MC-PDFT. We found that MC-PDFT calculations with small and large active spaces yield similar MECP energies.)

A vibrational analysis at the MECPs confirms that they are true minima on the multidimensional crossing seam with a single imaginary frequency in the effective Hessian, ${ }^{71}$ defined by a linear combination of the $T_{1}$ and $S_{0}$ Hessians. The collinear gradients of the $T_{1}$ and $S_{0}$ states at the located MECPs show that the crossing states conform to the Landau-Zener type of avoided crossing in the spin-adiabatic representation. Because internal energies in the $\mathrm{T}_{1}$ state range from 0.76 to $1.23 \mathrm{eV}$ for excitation wavelengths of $294-264 \mathrm{~nm}$, the $\mathrm{T}_{1} \rightarrow \mathrm{S}_{0}$ ISC can be mediated by any of these four MECPs.

The crossing points A and B are the lowest accessible MECPs lying only 0.41 and $0.42 \mathrm{eV}$ above the diallylic minimum. They share similar quinoidal skeletons with the ortho carbon being heavily distorted from the plane of the phenyl ring and differ by the inversion of the amino group 
(see Figure 2: A vs B structures). The prefulvene-like crossing point $\mathrm{C}$ lying at $0.95 \mathrm{eV}$ is the least accessible of the four MECPs. Unlike the amino group in the other MECPs, the amino group at crossing point $\mathrm{C}$ does not have a pyramidal shape residing within the plane formed by the ipso and ortho carbon atoms. The $\mathrm{C}_{s}$ symmetrical crossing point $\mathrm{D}$ at $0.45 \mathrm{eV}$ is characterized by the distorted ipso-carbon and $\mathrm{C}-\mathrm{N}$ bond being perpendicular to the plane of the five-membered ring excluding the ipso-carbon. The symmetry of the MECPs defines the degeneracy of the corresponding reaction paths. ${ }^{100}$ For the ISC paths through the A, B, and C MECPs, the degeneracy $f$ is equal to 2 due to stereoisomers. The MECP D has a plane of symmetry and therefore corresponding ISC path is non-degenerate and $f$ is equal to 1 .

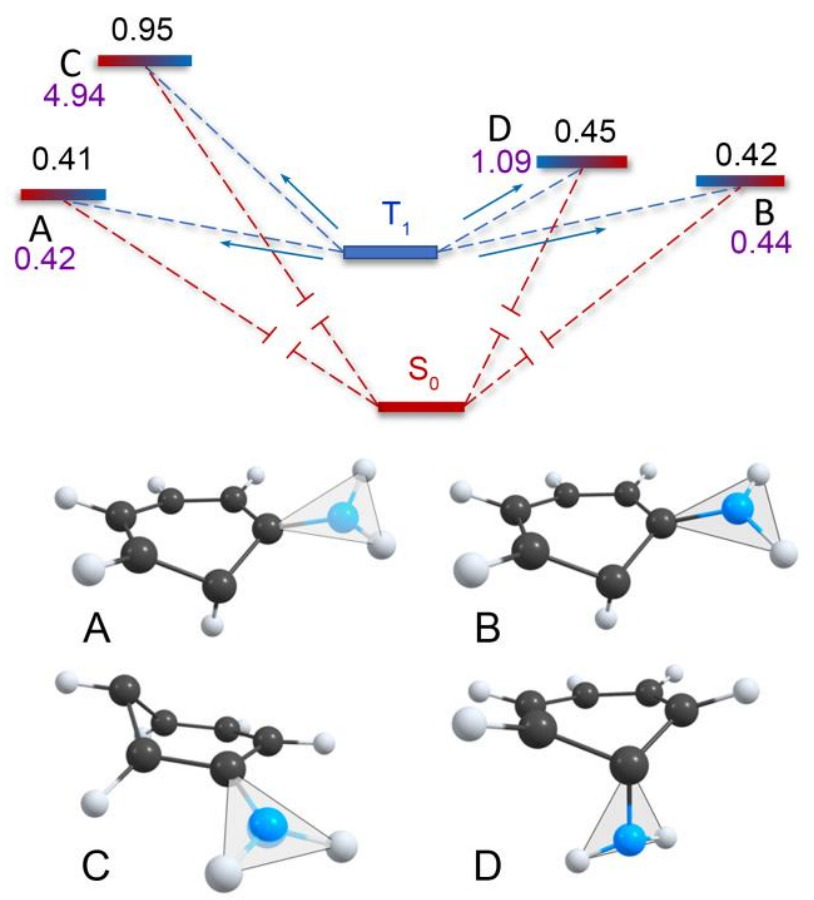

Figure 3. Schematic paths of the $\mathrm{T}_{1} \rightarrow \mathrm{S}_{0}$ ISC mediated by the minimum energy crossing points between $\mathrm{T}_{1}$ and $\mathrm{S}_{0}$ states in aniline. The ZPE-inclusive energies (black, above the line) are in $\mathrm{eV}$, and the spin-orbit coupling constants (magenta, below the line) are in $\mathrm{cm}^{-1}$.

One potential problem that may arise when calculating energies at the MECPs is the coupling between the crossing states and the close-lying excited states. If there is a conical intersection next to the MECP, the accurate prediction of the MECP energy may require a multistate multireference treatment. To address this issue and calculate the electronic energy gaps, we 
used XMS(3)-PDFT, where state-averaging was limited to the three lowest states within the given spin-manifold. The $\mathrm{T}_{4}$ excited state was excluded from averaging as it lies $\sim 2.23 \mathrm{eV}$ above the $\mathrm{T}_{1}$ state and therefore cannot contribute to the radiationless relaxation. As reported in Tables 3 and 4, the high-lying triplet states $\mathrm{T}_{2}$ and $\mathrm{T}_{3}$ are well-separated from $\mathrm{T}_{1}$ across all geometries, except for the Franck-Condon region where the $\mathrm{T}_{1}-\mathrm{T}_{2}$ and $\mathrm{T}_{1}-\mathrm{T}_{3}$ gaps are significantly reduced to 0.11 and $0.75 \mathrm{eV}$, respectively. These values agree well with the previously calculated vertical gaps of 0.08 and $0.66 \mathrm{eV}$ using single-point MS(4)-CASPT2 $(10,12)$ calculations at the geometries optimized with CASSCF(10,12)/ANO-S. ${ }^{101}$

Table 4 shows that the separation within the singlet and within the triplet manifolds is large for all MECPs, except for the crossing point $C$ where the $S_{0}-S_{1}$ splitting is only $0.37 \mathrm{eV}$. In fact, the prefulvene-like crossing point $C$ is reminiscent of the $S_{1}\left({ }^{1} \pi \pi^{*}\right)-S_{0} C I$ found in the work by Sala et al. ${ }^{88}$ However, the close-lying $S_{1}$ state is not accessible at the energies of interest, and therefore the two-state-crossing model for $\mathrm{T}_{1} \rightarrow \mathrm{S}_{0}$ ISC is still satisfied. The computed spin-orbit coupling constants mediating transitions between $\mathrm{T}_{1}$ and $\mathrm{S}_{0}$ states are small with the largest magnitude being $5 \mathrm{~cm}^{-1}$ at MECP C (see Table 5). Although this could facilitate ISC, the large ISC barrier of 0.96 $\mathrm{eV}$ makes transitions through crossing point $\mathrm{C}$ kinetically irrelevant.

Table 3. XMS-tPBE energies $(\mathrm{eV})$ of the triplet states relative to $\mathrm{T}_{1}$ at the four geometries as computed with the $(14,14)$ active space.

\begin{tabular}{lcccc}
\hline \hline & $\begin{array}{l}\text { Franck- } \\
\text { Condon }\end{array}$ & N-H TS & Diallylic & Quinoid \\
\cline { 2 - 5 } $\mathrm{T}_{1}$ & 0 & 0 & 0 & 0 \\
$\mathrm{~T}_{2}$ & 0.11 & 1.79 & 1.34 & 1.61 \\
$\mathrm{~T}_{3}$ & 0.75 & 2.13 & 2.02 & 2.79 \\
\hline \hline
\end{tabular}

Table 4. XMS-tPBE energies at the minimum energy crossing points $(\mathrm{eV})$ relative to the crossing energy of $S_{0}$ and $T_{1}$, computed with the $(14,14)$ active space.

\begin{tabular}{lcccc}
\hline & A & B & C & D \\
\hline $\mathrm{T}_{1}$ & 0 & 0 & 0 & 0 \\
$\mathrm{~T}_{2}$ & 2.33 & 2.31 & 2.13 & 2.19 \\
$\mathrm{~T}_{3}$ & 4.17 & 4.16 & 2.92 & 3.85 \\
$\mathrm{~S}_{0}$ & 0 & 0 & 0 & 0 \\
$\mathrm{~S}_{1}$ & 1.48 & 1.45 & 0.37 & 1.00 \\
$\mathrm{~S}_{2}$ & 2.63 & 2.62 & 2.47 & 2.63 \\
\hline \hline
\end{tabular}


Table 5. Spin-orbit coupling constants $\left(\mathrm{cm}^{-1}\right)$ at the $T_{1} / \mathrm{S}_{0}$ minimum-energy crossing points computed with the $(14,14)$ active space.

\begin{tabular}{ccccc}
\hline \hline & A & B & C & D \\
\hline SOC & 0.42 & 0.44 & 4.94 & 1.09 \\
\hline \hline
\end{tabular}

The ISC rates computed using Eqs. (1) and (2) for dynamics and tPBE(14,14)/def2-TZVP for electronic structure are shown in Figure 4. Since the A, B, and C MECPs have stereoisomers, the corresponding ISC paths are doubly degenerate. Despite the reasonable agreement of the rate constants at the low internal energies in the $\mathrm{T}_{1}$ state and nonradiative relaxation rates derived from the experiment of Knee and Johnson, the absolute values are extremely sensitive to the magnitude of spin-orbit coupling. For example, an error of only $0.5 \mathrm{~cm}^{-1}$ in spin-orbit coupling at the crossing point D increases the ISC rate constant by one order of magnitude. Thus, it is more appropriate to consider the overall trends than the absolute rates of ISC. While the computed ISC rate constants plotted in Figure 4 show a very weak dependence upon excitation energy, the experimental rates increase by more than one order of magnitude from internal energy of $0.76 \mathrm{eV}$ to $1.23 \mathrm{eV}$. Therefore, regardless of the agreement between the experimental and predicted ISC rates, we conclude that the ISC cannot be solely responsible for the $\mathrm{T}_{1}$ decay.

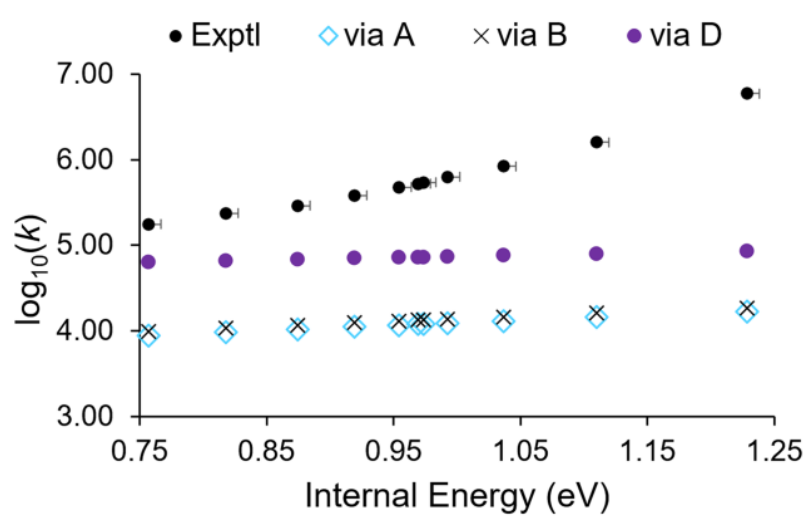

Figure 4. Experimental nonradiative rate constants and computed $\mathrm{T}_{1} \rightarrow \mathrm{S}_{0}$ intersystem rate constants for the reaction paths proceeding through the minimum energy crossing points $\mathrm{A}, \mathrm{B}$, and $\mathrm{D}$ optimized with $\mathrm{tPBE}(14,14)$. Positive error bars for experimental rate constants account for the possibly underestimated internal energies in the $T_{1}$ state. The zero of energy for this figure is the zero-point energy level of $\mathrm{T}_{1}$. 
The alternative explanation for the slow decay of the $T_{1}$ state is dissociation to the anilino radical, $\mathrm{C}_{6} \mathrm{H}_{5} \mathrm{NH}_{2} \rightarrow \mathrm{C}_{6} \mathrm{H}_{5} \mathrm{NH} \cdot+\mathrm{H} \cdot$. Upon elongation of the $\mathrm{N}-\mathrm{H}$ bond, the $\sigma(\mathrm{N}-\mathrm{H})$ and $\sigma^{*}(\mathrm{~N}-\mathrm{H})$ molecular orbitals become singly populated, which breaks the local symmetry of the amino group (see Figure S1 in the SI). Since the small active space does not have orbitals appropriate for describing the fission of $\mathrm{N}-\mathrm{H}$ bond, it cannot be used to evaluate the dissociation rates.

We therefore employed the large $(14,14)$ active space to account for the bond breaking, the planarization of the anilino radical, and the shortening of the $\mathrm{C}-\mathrm{N}$ bond upon N-H stretching. This active space includes $\sigma(\mathrm{N}-\mathrm{H})$ and $\sigma^{*}(\mathrm{~N}-\mathrm{H})$ molecular orbitals for each $\mathrm{N}-\mathrm{H}$ bond, not only to ensure the correct $C_{\mathrm{s}}$ symmetry in the quinoid conformer but also to provide a balanced description of the $\mathrm{N}-\mathrm{H}$ transition state. For completeness, we also report the N-H barrier with the $(12,12)$ active space where only one set of $\sigma(\mathrm{N}-\mathrm{H})$ and $\sigma^{*}(\mathrm{~N}-\mathrm{H})$ molecular orbitals is used. We found a transition state lying $0.68 \mathrm{eV}$ above the diallylic minimum with $\operatorname{tPBE}(12,12)$. Expanding the active space to $(14,14)$, this barrier increases to $0.76 \mathrm{eV}$. In a similar way, the ftPBE N-H dissociation barrier increases from 0.73 to $0.80 \mathrm{eV}$ when expanding the active space from $(12,12)$ to $(14,14)$. The minimum energy path calculations suggest that the $\mathrm{N}-\mathrm{H}$ transition state is connected to the anilino radical on one side and to the diallylic minimum on the other side. The potential energy curves shifted by the difference in ZPE in diallylic equilibrium and transition state are illustrated in Figure 5. 

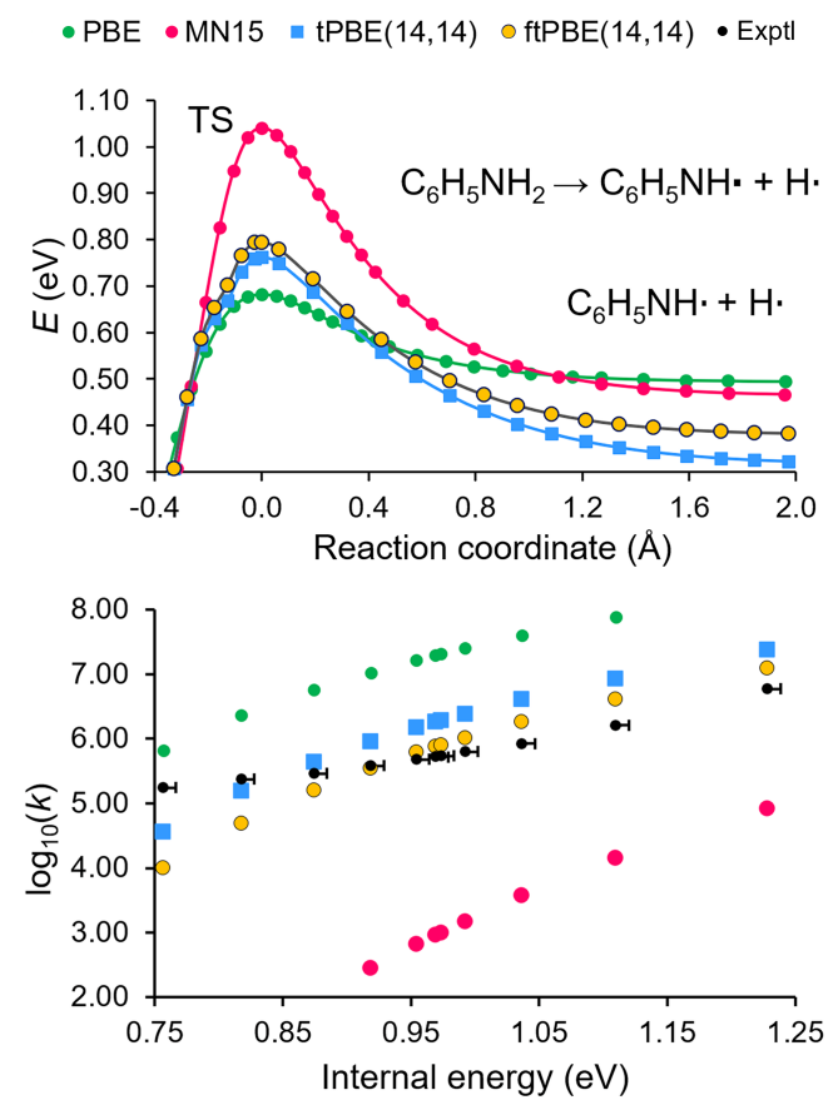

Figure 5. Upper panel: Potential energy curves for aniline dissociation in the $\mathrm{T}_{1}$ state. Lower panel: Experimental nonradiative rate constants (black) vs computed rate constants for aniline dissociation in the $T_{1}$ state. Positive error bars account for possibly underestimated internal energy in the $T_{1}$ state. The zero of energy for this figure is the zero-point energy level of $\mathrm{T}_{1}$.

When compared to the close-lying $\operatorname{tPBE}(14,14)$ and $\operatorname{ftPBE}(14,14)$ references, the PBE barrier is somewhat underestimated by $\sim 0.12 \mathrm{eV}$, whereas the barrier predicted by MN15 is overestimated by $\sim 0.25 \mathrm{eV}$. These differences can be attributed to the multiconfigurational nature of the transition state that is challenging for single-reference KS theory methods. The reverse barrier for the N-H exit channel predicted by all four methods is due to the conjugation between the nitrogen and the phenyl ring taking place in the completely planar anilino radical. Using Eq (2), we calculated the microcanonical rate constants for aniline dissociation in the $\mathrm{T}_{1}$ state, and these are summarized in Table 6 and depicted on a logarithmic scale in the lower panel of Figure 5. Note that the dissociation path is doubly-degenerate because of the two equivalent hydrogen atoms in the amino group. ${ }^{100}$ 
Table 6. Internal energies, experimental lifetimes and nonradiative rate constants for $\mathrm{T}_{1}$ decay, and computed rate constants for aniline dissociation in $\mathrm{T}_{1}$ state.

\begin{tabular}{|c|c|c|c|c|c|c|}
\hline \multirow{2}{*}{$\begin{array}{c}\text { Internal energy } \\
(\mathrm{eV})\end{array}$} & \multirow{2}{*}{$\begin{array}{l}\text { Lifetime (ms) } \\
\text { Exptl }\end{array}$} & \multicolumn{5}{|c|}{ Rate constant $\left(\mathrm{s}^{-1}\right)$, } \\
\hline & & Exptl $^{a}$ & PBE & MN15 & $\operatorname{tPBE}(14,14)$ & $\mathrm{ftPBE}(14,14)$ \\
\hline 0.76 & 5.65 & $1.76 \times 10^{5}$ & $6.5 \times 10^{5}$ & 2.8 & $3.6 \times 10^{4}$ & $9.9 \times 10^{3}$ \\
\hline 0.82 & 4.20 & $2.38 \times 10^{5}$ & $2.3 \times 10^{6}$ & $1.8 \times 10^{1}$ & $1.5 \times 10^{5}$ & $4.9 \times 10^{4}$ \\
\hline 0.87 & 3.44 & $2.90 \times 10^{5}$ & $5.7 \times 10^{6}$ & $8.9 \times 10^{1}$ & $4.4 \times 10^{5}$ & $1.6 \times 10^{5}$ \\
\hline 0.92 & 2.61 & $3.83 \times 10^{5}$ & $1.0 \times 10^{7}$ & $2.8 \times 10^{2}$ & $8.9 \times 10^{5}$ & $3.4 \times 10^{5}$ \\
\hline 0.95 & 2.09 & $4.79 \times 10^{5}$ & $1.6 \times 10^{7}$ & $6.5 \times 10^{2}$ & $1.5 \times 10^{6}$ & $6.1 \times 10^{5}$ \\
\hline 0.969 & 1.90 & $5.26 \times 10^{5}$ & $1.9 \times 10^{7}$ & $9.1 \times 10^{2}$ & $1.8 \times 10^{6}$ & $7.5 \times 10^{5}$ \\
\hline 0.974 & 1.83 & $5.46 \times 10^{5}$ & $2.0 \times 10^{7}$ & $9.9 \times 10^{2}$ & $1.9 \times 10^{6}$ & $7.9 \times 10^{5}$ \\
\hline 0.99 & 1.63 & $6.30 \times 10^{5}$ & $2.5 \times 10^{7}$ & $1.5 \times 10^{3}$ & $2.4 \times 10^{6}$ & $1.0 \times 10^{6}$ \\
\hline 1.04 & 1.19 & $8.38 \times 10^{5}$ & $3.9 \times 10^{7}$ & $3.7 \times 10^{3}$ & $4.0 \times 10^{6}$ & $1.8 \times 10^{6}$ \\
\hline 1.11 & 0.62 & $1.61 \times 10^{6}$ & $7.6 \times 10^{7}$ & $1.4 \times 10^{4}$ & $8.5 \times 10^{6}$ & $4.1 \times 10^{6}$ \\
\hline 1.23 & 0.17 & $5.94 \times 10^{6}$ & $1.8 \times 10^{8}$ & $8.1 \times 10^{4}$ & $2.3 \times 10^{7}$ & $1.2 \times 10^{7}$ \\
\hline
\end{tabular}

${ }^{a}$ Experimental rate constants are obtained as the reciprocals of the $\mathrm{T}_{1}$ lifetimes.

The tPBE and ftPBE rate constants agree within one order of magnitude with the experimental rates derived from the lifetimes measured by Knee and Johnson, suggesting that the radiationless relaxation in the $T_{1}$ state proceeds through the dissociation of aniline into the anilino radical accompanied by release of a hydrogen atom: $\mathrm{C}_{6} \mathrm{H}_{5} \mathrm{NH}_{2} \rightarrow \mathrm{C}_{6} \mathrm{H}_{5} \mathrm{NH} \cdot+\mathrm{H} \cdot$. The deviation of the first two excitation energies is probably due to the underestimated tunneling computed due to using the zero-curvature approximation that neglects the reaction-path curvature. (The small- or large-curvature approximations would be more accurate; ${ }^{102,103}$ however, this would require a substantial computational effort in the absence at the present time of MC-PDFT analytical Hessians.) The competing dissociative mechanism resulting in the formation of phenyl and amino radicals, $\mathrm{C}_{6} \mathrm{H}_{5} \mathrm{NH}_{2} \rightarrow \mathrm{C}_{6} \mathrm{H}_{5} \cdot+\cdot \mathrm{NH}_{2}$, is not favorable because the larger $\mathrm{C}-\mathrm{N}$ dissociation barrier of $1.30 \mathrm{eV}$ compared to that of the amino hydrogen (Table 1). The alternative N-H dissociation of aniline in the $\mathrm{T}_{2}$ state does not occur because it requires the formation of the excited anilino radical lying at $2.78 \mathrm{eV}$ above the ground doublet state (as calculated by XMS(2)-tPBE(13,14)/def2TZVP), which is not accessible at the low excitation wave lengths of 294-270 nm (4.22-4.59 eV).

The formation of hydrogen photoproduct at wavelengths longer than $270 \mathrm{~nm}(4.59 \mathrm{eV}) \mathrm{was}$ in fact observed by King et al. by using $\mathrm{H}$ Rydberg atom photofragment translational 
spectroscopy. ${ }^{47}$ They reported a maximum of $\sim 0.37 \mathrm{eV}$ in the total kinetic energy release (TKER) spectra, which is in good agreement with the reverse barrier of $0.41 \mathrm{eV}$ predicted by ftPBE $(14,14)$. However, they also found that the TKER spectra extended up to $\sim 1.74 \mathrm{eV}$, in disagreement with the maximum kinetic energies calculated for the one-photon excitations. Indeed, the absorption of a single photon with the energy of $\sim 4.22 \mathrm{eV}$ (294 nm) restricts the hydrogen kinetic energy to be less than or equal to $0.29 \mathrm{eV}$. Therefore, they attributed hydrogen evolution to the multiphoton dissociation triggered by high-intensity laser pulses. Our calculations demonstrate that at least in part the TKER spectra measured by King et al. are due to the competing dissociation of aniline on the $\mathrm{T}_{1}$ PES. We believe that the computational evidence presented here for the hidden dissociation channel in the triplet state of aniline can also be important for understanding the relaxation dynamics of many other heteroaromatic compounds containing nitrogen and can complement the mechanisms of photoprotection in the biologically relevant molecules.

\section{CONCLUSIONS}

The remarkable photostability of many biologically relevant chromophores is commonly attributed to the efficient energy dissipation in the singlet manifold where the population of repulsive ${ }^{1} \pi \sigma^{*}$ excited state is followed by nonradiative relaxation back to the ground electronic state mediated by ${ }^{1} \pi \sigma^{*} / \mathrm{S}_{0}$ conical intersection. This redistribution of excitation energy into vibrational energy of the molecule prevents the detrimental effects of ultraviolet radiation. Recent experiments have provided evidence for triplet participation, but the mechanism has been unclear. In this paper we use theory to delineate such a mechanism for the case of aniline. In particular, by using recently developed electronic structure capabilities combined with calculations of both spinchanging and spin-conserving mechanisms, we demonstrate that the population loss of aniline in the triplet manifold is dominated by spin-conserving $\mathrm{C}_{6} \mathrm{H}_{5} \mathrm{NH}_{2} \rightarrow \mathrm{C}_{6} \mathrm{H}_{5} \mathrm{NH} \cdot+\mathrm{H} \cdot$ dissociation in the $\mathrm{T}_{1}$ state, and we explain the long nonradiative lifetimes of the $\mathrm{T}_{1}$ state at the excitation wavelengths of 294-264 nm.

The simulations have been carried out using statistical theories for dynamics coupled with the MC-PDFT method for electronic structure, the latter being used for the first time to locate the minimum-energy crossing points between states of different spin, in particular between the excited 
triplet and the ground singlet states involved in $\mathrm{T}_{1} \rightarrow \mathrm{S}_{0}$ ISC. We have shown that the ISC to the ground state cannot explain the puzzling radiationless lifetimes of the $\mathrm{T}_{1}$ state produced from the optically populated $S_{1}$ vibronic states at the low excitation energies from $4.22 \mathrm{eV}$ (294 nm) to $4.70 \mathrm{eV}(264 \mathrm{~nm})$. While the measured microsecond lifetimes exhibit 33-fold decrease upon increase in the excitation energy, the predicted ISC rates show weak energy dependence. This can be attributed to the small energetic barrier required to reach the multidimensional crossing seam where two states become degenerate. Thus, the ISC rates are limited by the weak spin-orbit coupling between $T_{1}$ and $S_{0}$ states that has been found to be on the order of a few wave numbers. On the other hand, we have shown that the experimental long-lived lifetimes are in good agreement with the computed rate constants for dissociation in the lowest triplet state, $\mathrm{C}_{6} \mathrm{H}_{5} \mathrm{NH}_{2} \rightarrow \mathrm{C}_{6} \mathrm{H}_{5} \mathrm{NH} \cdot+\mathrm{H} \cdot$, which is more efficient than dissociation in the $\mathrm{T}_{2}$ state or dissociation into phenyl and amino radicals, $\mathrm{C}_{6} \mathrm{H}_{5} \mathrm{NH}_{2} \rightarrow \mathrm{C}_{6} \mathrm{H}_{5} \cdot+\cdot \mathrm{NH}_{2}$. Our calculations demonstrate that even excitations slightly above the $S_{1}$ origin lead to hydrogen-atom release through the exit channel on the $T_{1}$ PES competing to the ${ }^{1} \pi \sigma^{*}$ dissociation channel. Similar behavior can be expected for the other heteroaromatic molecules with a remarkable example being the recently discovered triplet-mediated dissociation of N-methylpyrrole. ${ }^{104}$

Another aspect of this work is that we have shown that MC-PDFT is a valuable tool to probe the kinetics of excited electronic states, which are often inherently multiconfigurational and therefore challenging for single-reference methods. We believe that, with the now-available analytical gradients, MC-PDFT provides a natural choice for location of the minimum energy crossing points between the lowest excited states of different spin. The case of triplet aniline shows how both spin-allowed and spin-forbidden relaxation mechanisms can be treated by MC-PDFT including excited-state optimizations. Moreover, the ongoing development of multi-state MCPDFT gradients will open the door to molecular dynamics simulations of photochemical reactions.

\section{Appendix: Effective Hessian at the MECP}

The effective Hessian can be derived by using Lagrange multipliers. The Lagrangian is

$$
L(\mathbf{r}, \lambda)=E_{1}(\mathbf{r})-\lambda\left[E_{1}(\mathbf{r})-E_{2}(\mathbf{r})\right],
$$


where $E_{1}(\mathbf{r})$ and $E_{2}(\mathbf{r})$ are the energies of two crossing states, $\mathbf{r}$ is the vector of nuclear coordinates, and $\lambda$ is the Lagrange multiplier. At the MECP,

$$
\begin{aligned}
& L\left(\mathbf{r}_{\mathrm{MECP}}, \lambda_{\mathrm{MECP}}\right)=E_{1}\left(\mathbf{r}_{\mathrm{MECP}}\right)=E_{2}\left(\mathbf{r}_{\mathrm{MECP}}\right)=E_{\mathrm{MECP}}, \\
& \nabla_{\mathrm{r}} L\left(\mathbf{r}_{\mathrm{MECP}}, \lambda_{\mathrm{MECP}}\right)=\mathbf{g}_{1}-\lambda_{\mathrm{MECP}}\left(\mathbf{g}_{1}-\mathbf{g}_{2}\right)=0,
\end{aligned}
$$

where $\mathbf{g}_{1}$ and $\mathbf{g}_{2}$ are the gradients of the two crossing states. The expansion of energy $E_{1}$ at the MECP in the Taylor series up to the second order with a displacement $\Delta \mathbf{S}$ along the seam is equivalent to the expansion of the Lagrangian $\nabla_{\mathrm{r}} L$

$$
\begin{aligned}
& L\left(\mathbf{r}_{\mathrm{MECP}}+\Delta \mathbf{s}, \lambda_{\mathrm{MECP}}\right)=L\left(\mathbf{r}_{\mathrm{MECP}}, \lambda_{\mathrm{MECP}}\right)+ \\
& \Delta \mathbf{s}^{\mathrm{T}} \nabla_{\mathrm{r}} L\left(\mathbf{r}_{\mathrm{MECP}}, \lambda_{\mathrm{MECP}}\right)+\frac{1}{2} \Delta \mathbf{s}^{\mathrm{T}} \nabla_{\mathrm{r}}^{2} L\left(\mathbf{r}_{\mathrm{MECP}}, \lambda_{\mathrm{MECP}}\right) \Delta \mathbf{s}
\end{aligned}
$$

The second term is zero by eq. S3, and consequently the expansion of $E_{1}$ can be written as

$$
\begin{aligned}
& E_{1}\left(\mathbf{r}_{\mathrm{MECP}}+\Delta \mathbf{s}\right)=L\left(\mathbf{r}_{\mathrm{MECP}}+\Delta \mathbf{s}, \lambda_{\mathrm{MECP}}\right)= \\
& L\left(\mathbf{r}_{\mathrm{MECP}}, \lambda_{\mathrm{MECP}}\right)+\frac{1}{2} \Delta \mathbf{s}^{\mathrm{T}} \nabla_{\mathrm{r}}^{2} L\left(\mathbf{r}_{\mathrm{MECP}}, \lambda_{\mathrm{MECP}}\right) \Delta \mathbf{s}=E_{\mathrm{MECP}}+\frac{1}{2} \Delta \mathbf{s}^{\mathrm{T}} \mathbf{H}_{\mathrm{eff}} \Delta \mathbf{s}^{\prime}
\end{aligned}
$$

where the effective Hessian, $\mathbf{H}_{\text {eff }}$ is the matrix of the second derivatives of the Lagrangian with respect to $\mathbf{r}$. The explicit form of $\mathbf{H}_{\text {eff }}$, can be found by differentiating eq. S1:

$$
\mathbf{H}_{\mathrm{eff}}=\nabla_{\mathrm{r}}^{2} E_{1}(\mathbf{r})-\lambda_{\mathrm{MECP}}\left[\nabla_{\mathrm{r}}^{2} E_{1}(\mathbf{r})-\nabla_{\mathrm{r}}^{2} E_{2}(\mathbf{r})\right]=\mathbf{H}_{1}-\lambda_{\mathrm{MECP}}\left(\mathbf{H}_{1}-\mathbf{H}_{2}\right)
$$

Solving eq. S3 for the Lagrange multiplier, one obtains 


$$
\lambda_{\mathrm{MECP}}=\frac{\Delta \mathbf{g}^{\mathrm{T}} \mathbf{g}_{1}}{|\Delta \mathbf{g}|^{2}}=\frac{\left|\mathbf{g}_{1}\right| \cos (\theta)}{|\Delta \mathbf{g}|}
$$

where $\Delta \mathbf{g}=\mathbf{g}_{1}-\mathbf{g}_{2}$ is the difference gradient orthogonal to the crossing seam and $\theta$ is the angle between vectors $\Delta \mathbf{g}$ and $\mathbf{g}_{1}$. Substituting (S7) into (S6) yields

$$
\mathbf{H}_{\text {eff }}=\frac{\left|\mathbf{g}_{1}\right| \cos (\theta) \mathbf{H}_{2}+\left[|\Delta \mathbf{g}|-\left|\mathbf{g}_{1}\right| \cos (\theta)\right] \mathbf{H}_{1}}{|\Delta \mathbf{g}|}
$$

Since vectors $\mathbf{g}_{1}$ and $\mathbf{g}_{2}$ are both perpendicular to the seam, they are either parallel or antiparallel, and the angle $\theta$ between $\mathbf{g}_{1}$ and $\Delta \mathbf{g}$ must therefore be $0^{\circ}$ or $180^{\circ}$. Therefore Eq. S8 becomes

$$
\begin{gathered}
\mathbf{H}_{\text {eff }}=\frac{\left|\mathbf{g}_{1}\right| \mathbf{H}_{2}+\left|\mathbf{g}_{2}\right| \mathbf{H}_{1}}{\left|\mathbf{g}_{1}\right|+\left|\mathbf{g}_{2}\right|}, \text { if } \mathbf{g}_{1}^{\mathrm{T}} \mathbf{g}_{2}<0,\left\{\begin{array}{l}
\left|\mathbf{g}_{1}\right|<\left|\mathbf{g}_{2}\right|,|\Delta \mathbf{g}|=\left|\mathbf{g}_{1}\right|+\left|\mathbf{g}_{2}\right|, \cos (\theta)=1 \\
\left|\mathbf{g}_{1}\right|>\left|\mathbf{g}_{2}\right|,|\Delta \mathbf{g}|=\left|\mathbf{g}_{1}\right|+\left|\mathbf{g}_{2}\right|, \cos (\theta)=1
\end{array}\right. \\
\mathbf{H}_{\text {eff }}=\frac{\left|\mathbf{g}_{1}\right| \mathbf{H}_{2}-\left|\mathbf{g}_{2}\right| \mathbf{H}_{1}}{\left|\mathbf{g}_{1}\right|-\left|\mathbf{g}_{2}\right|}, \text { if } \mathbf{g}_{1}^{\mathrm{T}} \mathbf{g}_{2}>0,\left\{\begin{array}{l}
\left|\mathbf{g}_{1}\right|<\left|\mathbf{g}_{2}\right|,|\Delta \mathbf{g}|=\left|\mathbf{g}_{2}\right|-\left|\mathbf{g}_{1}\right|, \cos (\theta)=-1 \\
\left|\mathbf{g}_{1}\right|>\left|\mathbf{g}_{2}\right|,|\Delta \mathbf{g}|=\left|\mathbf{g}_{1}\right|-\left|\mathbf{g}_{2}\right|, \cos (\theta)=1
\end{array}\right.
\end{gathered}
$$

which can be written more succinctly as

$$
\mathbf{H}_{\text {eff }}=\frac{\left|\mathbf{g}_{1}\right| \mathbf{H}_{2} \pm\left|\mathbf{g}_{2}\right| \mathbf{H}_{1}}{\left|\mathbf{g}_{1}\right| \pm\left|\mathbf{g}_{2}\right|}
$$

where the plus sign is used if the intersection is peaked $\left(\mathbf{g}_{1}^{\mathrm{T}} \mathbf{g}_{2}<0\right)$, and the minus sign is used if the intersection is sloped $\left(\mathbf{g}_{1}^{\mathrm{T}} \mathbf{g}_{2}>0\right)$. In the case of aniline, the dot product between vectors $\mathbf{g}_{1}$ and $\mathbf{g}_{2}$ is positive for all MECPs; thus the negative sign is used. 
The vibrational frequencies at the MECP can be obtained by diagonalizing the mass-weighted effective Hessian where 7 degrees of freedom are projected out to eliminate translational and rotational contributions together with the contribution of the reaction coordinate. The projected effective Hessian is

$$
\begin{gathered}
\mathbf{H}_{\mathrm{eff}}^{\prime}=\left(\mathbf{I}_{3 N}-\mathbf{P}\right) \mathbf{H}_{\mathrm{eff}}^{\mathrm{mw}}\left(\mathrm{I}_{3 N}-\mathbf{P}\right), \\
\mathbf{P}=\mathbf{P}_{\mathrm{tr}}+\mathbf{P}_{\mathrm{rot}}+\mathbf{P}_{\mathrm{rc}}, \\
\left\{\mathbf{H}_{\mathrm{eff}}^{\mathrm{mw}}\right\}_{i j}=\frac{\left\{\mathbf{H}_{\mathrm{eff}}\right\}_{i j}}{\sqrt{m_{i} m_{j}}},
\end{gathered}
$$

where $m_{\mathrm{i}}$ is the mass of the atom associated with coordinate $i, \mathbf{I}$ is the identity matrix and $\mathbf{P}$ is the projector matrix with elements defined in ref ${ }^{105}$. The reduced mass of the reaction coordinate at the MECP can be found by projecting the mass-weighted effective Hessian onto the reaction coordinate

$$
\begin{aligned}
& \mathbf{H}_{\mathrm{eff}}^{\prime \prime}=\mathbf{P}_{\mathrm{rc}} \mathbf{H}_{\mathrm{eff}}^{\mathrm{mw}} \mathbf{P}_{\mathrm{rc}}, \\
& \mathbf{P}_{\mathrm{rc}}=\mathbf{u} \mathbf{u}^{\mathrm{T}}, \mathbf{u}=\frac{\Delta \mathbf{g}}{|\Delta \mathbf{g}|} .
\end{aligned}
$$

The subsequent diagonalization of the projected effective Hessian yields a single nonzero eigenvalue with the corresponding unitless eigenvector $\mathbf{v}^{\mathrm{mw}}$, which we normalize to unity. This is transformed to the eigenvector of the unweighted Hessian by dividing each element by $\sqrt{m_{i}}$

$$
v_{i}=v_{i}^{\mathrm{mw}} / \sqrt{m_{i}}
$$

The reduced mass of the reaction coordinate is then calculated as

$$
\mu=\left(\mathbf{v}^{\mathrm{T}} \mathbf{v}\right)^{-1}
$$




\section{ASSOCIATED CONTENT}

Supporting information includes natural orbitals and occupation numbers, harmonic vibrational frequencies, Cartesian coordinates of optimized structures.

\section{AUTHOR INFORMATION}

Aleksandr O. Lykhin, Department of Chemistry, Pritzker School of Molecular Engineering, The James Franck Institute and Chicago Center for Theoretical Chemistry, The University of Chicago, Chicago, IL 60637, United States

orcid.org/0000-0002-9366-5866

Email: 1ykhin@uchucago.edu

Donald G. Truhlar, Department of Chemistry, Chemical Theory Center, and Minnesota Supercomputing Institute, University of Minnesota, 207 Pleasant Street SE, Minneapolis, Minnesota 55455, United States

orcid.org/0000-0002-7742-7294

Email: truhlar@umn.edu

Laura Gagliardi, Department of Chemistry, Pritzker School of Molecular Engineering, The James Franck Institute and Chicago Center for Theoretical Chemistry, The University of Chicago, Chicago, IL 60637, United States

orcid.org/0000-0001-5227-1396

Email: lgagliardi@uchicago.edu

\section{Notes}

The authors declare no competing financial interest. 


\section{ACKNOWLEDGMENTS}

This work was supported by the Air Force Office of Scientific Research by Grant No. FA9550-16$1-0134$.

\section{REFERENCES}

(1) Oruganti, B.; Pál Kalapos, P.; Bhargav, V.; London, G.; Durbeej, B. Photoinduced Changes in Aromaticity Facilitate Electrocyclization of Dithienylbenzene Switches. J. Am. Chem. Soc. 2020, 142 (32), 13941-13953. https://doi.org/10.1021/jacs.0c06327.

(2) Schulz, M.; Hagmeyer, N.; Wehmeyer, F.; Lowe, G.; Rosenkranz, M.; Seidler, B.; Popov, A.; Streb, C.; Vos, J. G.; Dietzek, B. Photoinduced Charge Accumulation and Prolonged Multielectron Storage for the Separation of Light and Dark Reaction. J. Am. Chem. Soc. 2020, 142 (37), 15722-15728. https://doi.org/10.1021/jacs.0c03779.

(3) Harlang, T. C. B.; Liu, Y.; Gordivska, O.; Fredin, L. A.; Ponseca, C. S.; Huang, P.; Chábera, P.; Kjaer, K. S.; Mateos, H.; Uhlig, J.; Lomoth, R.; Wallenberg, R.; Styring, S.; Persson, P.; Sundström, V.; Wärnmark, K. Iron Sensitizer Converts Light to Electrons with 92\% Yield. Nat. Chem. 2015, 7 (11), 883-889. https://doi.org/10.1038/nchem.2365.

(4) Brennaman, M. K.; Dillon, R. J.; Alibabaei, L.; Gish, M. K.; Dares, C. J.; Ashford, D. L.; House, R. L.; Meyer, G. J.; Papanikolas, J. M.; Meyer, T. J. Finding the Way to Solar Fuels with Dye-Sensitized Photoelectrosynthesis Cells. J. Am. Chem. Soc. 2016, 138 (40), 13085-13102. https://doi.org/10.1021/jacs.6b06466.

(5) Zhao, Q.; Huang, H.; Li, F. Phosphorescent Heavy-Metal Complexes for Bioimaging. Chem. Soc. Rev. 2011, 40 (5), 2508-2524. https://doi.org/10.1039/c0cs00114g.

(6) Ting, S. I.; Garakyaraghi, S.; Taliaferro, C. M.; Shields, B. J.; Scholes, G. D.; Castellano, F. N.; Doyle, A. G. ${ }^{3} \mathrm{~d}$-d Excited States of Ni(II) Complexes Relevant to Photoredox Catalysis: Spectroscopic Identification and Mechanistic Implications. J. Am. Chem. Soc. 2020, 142 (12), 5800-5810. https://doi.org/10.1021/jacs.0c00781.

(7) Mei, L.; Veleta, J. M.; Gianetti, T. L. Helical Carbenium Ion: A Versatile Organic Photoredox Catalyst for Red-Light-Mediated Reactions. J. Am. Chem. Soc. 2020, 142 (28), 12056-12061. https://doi.org/10.1021/jacs.0c05507.

(8) Minaev, B.; Baryshnikov, G.; Agren, H. Principles of Phosphorescent Organic Light Emitting Devices. Phys. Chem. Chem. Phys. 2014, 16 (5), 1719-1758. https://doi.org/10.1039/c3cp53806k.

(9) Zavaleta, A.; Lykhin, A. O.; Monteiro, J. H. S. K.; Uchida, S.; Bell, T. W.; De Bettencourt-Dias, A.; Varganov, S. A.; Gallucci, J. Full Visible Spectrum and White Light Emission with a Single, InputTunable Organic Fluorophore. J. Am. Chem. Soc. 2020. https://doi.org/10.1021/jacs.0c08182.

(10) Peng, P.; Marceau, C.; Villeneuve, D. M. Attosecond Imaging of Molecules Using High Harmonic 
Spectroscopy. Nat. Rev. Phys. 2019, 1 (2), 144-155. https://doi.org/10.1038/s42254-018-0015-1.

(11) Villeneuve, D. M. Attosecond Science. Contemp. Phys. 2018, 59 (1), 47-61. https://doi.org/10.1080/00107514.2017.1407093.

(12) Jiang, L.; Wang, A. D.; Li, B.; Cui, T. H.; Lu, Y. F. Electrons Dynamics Control by Shaping Femtosecond Laser Pulses in Micro/Nanofabrication: Modeling, Method, Measurement and Application. Light Sci. Appl. 2018, 7 (2), 1-27. https://doi.org/10.1038/Isa.2017.134.

(13) Liu, Y.; Persson, P.; Sundström, V.; Wärnmark, K. Fe N-Heterocyclic Carbene Complexes as Promising Photosensitizers. Acc. Chem. Res. 2016, 49 (8), 1477-1485. https://doi.org/10.1021/acs.accounts.6b00186.

(14) Jäger, M.; Freitag, L.; González, L. Using Computational Chemistry to Design Ru Photosensitizers with Directional Charge Transfer. Coord. Chem. Rev. 2015, 304-305, 146-165. https://doi.org/10.1016/j.ccr.2015.03.019.

(15) Conti, I.; Cerullo, G.; Nenov, A.; Garavelli, M. Ultrafast Spectroscopy of Photoactive Molecular Systems from First Principles: Where We Stand Today and Where We Are Going. J. Am. Chem. Soc. 2020, 142 (38), 16117-16139. https://doi.org/10.1021/jacs.0c04952.

(16) Lischka, H.; Nachtigallová, D.; Aquino, A. J. A.; Szalay, P. G.; Plasser, F.; Machado, F. B. C.; Barbatti, M. Multireference Approaches for Excited States of Molecules. Chem. Rev. 2018, 118 (15), 72937361. https://doi.org/10.1021/acs.chemrev.8b00244.

(17) Park, J. W.; Al-Saadon, R.; MacLeod, M. K.; Shiozaki, T.; Vlaisavljevich, B. Multireference Electron Correlation Methods: Journeys along Potential Energy Surfaces. Chem. Rev. 2020, 120 (13), 58785909. https://doi.org/10.1021/acs.chemrev.9b00496.

(18) Teller, E. The Crossing of Potential Surfaces. J. Phys. Chem. 1937, 41 (1), 109-116. https://doi.org/10.1021/j150379a010.

(19) Gozem, S.; Melaccio, F.; Valentini, A.; Filatov, M.; Huix-Rotllant, M.; Ferré, N.; Frutos, L. M.; Angeli, C.; Krylov, A. I.; Granovsky, A. A.; Lindh, R.; Olivucci, M. Shape of Multireference, Equation-ofMotion Coupled-Cluster, and Density Functional Theory Potential Energy Surfaces at a Conical Intersection. J. Chem. Theory Comput. 2014, 10 (8), 3074-3084.

https://doi.org/10.1021/ct500154k.

(20) Levine, B. G.; Ko, C.; Quenneville, J.; Martínez, T. J. Conical Intersections and Double Excitations in Time-Dependent Density Functional Theory. Mol. Phys. 2006, 104 (5-7), 1039-1051. https://doi.org/10.1080/00268970500417762.

(21) Tuna, D.; Lefrancois, D.; Wolański, Ł.; Gozem, S.; Schapiro, I.; Andruniów, T.; Dreuw, A.; Olivucci, M. Assessment of Approximate Coupled-Cluster and Algebraic-Diagrammatic-Construction Methods for Ground- and Excited-State Reaction Paths and the Conical-Intersection Seam of a Retinal-Chromophore Model. J. Chem. Theory Comput. 2015, 11 (12), 5758-5781. https://doi.org/10.1021/acs.jctc.5b00022.

(22) Andersson, K.; Malmqvist, P. Å.; Roos, B. O. Second-Order Perturbation Theory with a Complete Active Space Self-Consistent Field Reference Function. J. Chem. Phys. 1992, 96 (2), 1218-1226. 
https://doi.org/10.1063/1.462209.

(23) Andersson, K.; Malmqvist, P. Å.; Roos, B. O.; Sadlej, A. J.; Wolinski, K. Second-Order Perturbation Theory with a CASSCF Reference Function. J. Phys. Chem. 1990, 94 (14), 5483-5488. https://doi.org/10.1021/j100377a012.

(24) Granovsky, A. A. Extended Multi-Configuration Quasi-Degenerate Perturbation Theory: The New Approach to Multi-State Multi-Reference Perturbation Theory. J. Chem. Phys. 2011, 134 (21). https://doi.org/10.1063/1.3596699.

(25) Shiozaki, T.; Gyroffy, W.; Celani, P.; Werner, H. J. Communication: Extended Multi-State Complete Active Space Second-Order Perturbation Theory: Energy and Nuclear Gradients. J. Chem. Phys. 2011, 135 (8). https://doi.org/10.1063/1.3633329.

(26) Celani, P.; Werner, H. J. Analytical Energy Gradients for Internally Contracted Second-Order Multireference Perturbation Theory. J. Chem. Phys. 2003, 119 (10), 5044-5057. https://doi.org/10.1063/1.1597672.

(27) Park, J. W.; Shiozaki, T. On-the-Fly CASPT2 Surface-Hopping Dynamics. J. Chem. Theory Comput. 2017, 13 (8), 3676-3683. https://doi.org/10.1021/acs.jctc.7b00559.

(28) Manni, G. L.; Carlson, R. K.; Luo, S.; Ma, D.; Olsen, J.; Truhlar, D. G.; Gagliardi, L. Multiconfiguration Pair-Density Functional Theory. J. Chem. Theory Comput. 2014, 10 (9), 3669-3680. https://doi.org/10.1021/ct500483t.

(29) Gagliardi, L.; Truhlar, D. G.; Manni, G. L.; Carlson, R. K.; Hoyer, C. E.; Bao, J. L. Multiconfiguration Pair-Density Functional Theory: A New Way to Treat Strongly Correlated Systems. Acc. Chem. Res. 2017, 50 (1), 66-73. https://doi.org/10.1021/acs.accounts.6b00471.

(30) Ghosh, S.; Verma, P.; Cramer, C. J.; Gagliardi, L.; Truhlar, D. G. Combining Wave Function Methods with Density Functional Theory for Excited States. Chem. Rev. 2018, 118 (15), 7249-7292. https://doi.org/10.1021/acs.chemrev.8b00193.

(31) Stoneburner, S. J.; Truhlar, D. G.; Gagliardi, L. Transition Metal Spin-State Energetics by MC-PDFT with High Local Exchange. J. Phys. Chem. A 2020, 124 (6), 1187-1195. https://doi.org/10.1021/acs.jpca.9b10772.

(32) Jacob, C. R.; Reiher, M. Spin in Density-Functional Theory. Int. J. Quantum Chem. 2012, 112 (23), 3661-3684. https://doi.org/10.1002/qua.24309.

(33) Stoneburner, S. J.; Truhlar, D. G.; Gagliardi, L. MC-PDFT Can Calculate Singlet-Triplet Splittings of Organic Diradicals. J. Chem. Phys. 2018, 148 (6), 064108. https://doi.org/10.1063/1.5017132.

(34) Bowman, D. N.; Jakubikova, E. Low-Spin versus High-Spin Ground State in Pseudo-Octahedral Iron Complexes. Inorg. Chem. 2012, 51 (11), 6011-6019. https://doi.org/10.1021/ic202344w.

(35) Reiher, M. Theoretical Study of the Fe(Phen $)_{2}(\mathrm{NCS})_{2}$ Spin-Crossover Complex with Reparametrized Density Functionals. Inorg. Chem. 2002, 41 (25), 6928-6935. https://doi.org/10.1021/ic025891I.

(36) Bao, J. L.; Gagliardi, L.; Truhlar, D. G. Self-Interaction Error in Density Functional Theory: An Appraisal. J. Phys. Chem. Lett. 2018, 9 (9), 2353-2358. 
https://doi.org/10.1021/acs.jpclett.8b00242.

(37) Sand, A. M.; Kidder, K. M.; Truhlar, D. G.; Gagliardi, L. Calculation of Chemical Reaction Barrier Heights by Multiconfiguration Pair-Density Functional Theory with Correlated Participating Orbitals. J. Phys. Chem. A 2019, 123 (45), 9809-9817. https://doi.org/10.1021/acs.jpca.9b08134.

(38) Sharma, P.; Bernales, V.; Truhlar, D. G.; Gagliardi, L. Valence $\pi \pi^{*}$ Excitations in Benzene Studied by Multiconfiguration Pair-Density Functional Theory. J. Phys. Chem. Lett. 2019, 10 (1), 75-81. https://doi.org/10.1021/acs.jpclett.8b03277.

(39) Sharma, P.; Bernales, V.; Knecht, S.; Truhlar, D. G.; Gagliardi, L. Density Matrix Renormalization Group Pair-Density Functional Theory (DMRG-PDFT): Singlet-Triplet Gaps in Polyacenes and Polyacetylenes. Chem. Sci. 2019, 10 (6), 1716-1723. https://doi.org/10.1039/c8sc03569e.

(40) Sand, A. M.; Hoyer, C. E.; Sharkas, K.; Kidder, K. M.; Lindh, R.; Truhlar, D. G.; Gagliardi, L. Analytic Gradients for Complete Active Space Pair-Density Functional Theory. J. Chem. Theory Comput. 2018, 14 (1), 126-138. https://doi.org/10.1021/acs.jctc.7b00967.

(41) Scott, T. R.; Hermes, M. R.; Sand, A. M.; Oakley, M. S.; Truhlar, D. G.; Gagliardi, L. Analytic Gradients for State-Averaged Multiconfiguration Pair-Density Functional Theory. J. Chem. Phys. 2020, 153 (1), 014106. https://doi.org/10.1063/5.0007040.

(42) Baker, L. A.; Marchetti, B.; Karsili, T. N. V.; Stavros, V. G.; Ashfold, M. N. R. Photoprotection: Extending Lessons Learned from Studying Natural Sunscreens to the Design of Artificial Sunscreen Constituents. Chem. Soc. Rev. 2017, 46 (12), 3770-3791. https://doi.org/10.1039/C7CS00102A.

(43) Middleton, C. T.; De La Harpe, K.; Su, C.; Law, Y. K.; Crespo-Hernández, C. E.; Kohler, B. DNA Excited-State Dynamics: From Single Bases to the Double Helix. Annu. Rev. Phys. Chem. 2009, 60, 217-239. https://doi.org/10.1146/annurev.physchem.59.032607.093719.

(44) Crespo-Hernández, C. E.; Cohen, B.; Hare, P. M.; Kohler, B. Ultrafast Excited-State Dynamics in Nucleic Acids. Chem. Rev. 2004, 104 (4), 1977-2019. https://doi.org/10.1021/cr0206770.

(45) Roberts, G. M.; Stavros, V. G. The Role of $\pi \sigma^{*}$ States in the Photochemistry of Heteroaromatic Biomolecules and Their Subunits: Insights from Gas-Phase Femtosecond Spectroscopy. Chem. Sci. 2014, 5 (5), 1698-1722. https://doi.org/10.1039/c3sc53175a.

(46) Latha, M. S.; Martis, J.; Shobha, V.; Shinde, R. S.; Bangera, S.; Krishnankutty, B.; Bellary, S.; Varughese, S.; Rao, P.; Kumar, B. R. N. Sunscreening Agents: A Review. J. Clin. Aesthet. Dermatol. 2013, 6 (1), 16-26.

(47) King, G. A.; Oliver, T. A. A.; Ashfold, M. N. R. Dynamical Insights into ${ }^{1} \pi \sigma^{*}$ State Mediated Photodissociation of Aniline. J. Chem. Phys. 2010, 132 (21), 214307.

https://doi.org/10.1063/1.3427544.

(48) Montero, R.; Conde, Á. P.; Ovejas, V.; Martínez, R.; Castaño, F.; Longarte, A. Ultrafast Dynamics of Aniline in the 294-234 nm Excitation Range: The Role of the $\Pi \sigma^{*}$ State. J. Chem. Phys. 2011, 135 (5), 054308. https://doi.org/10.1063/1.3615544.

(49) Spesyvtsev, R.; Kirkby, O. M.; Fielding, H. H. Ultrafast Dynamics of Aniline Following 269-238 nm 
Excitation and the Role of the $S_{2}\left(\pi 3 s / \pi \sigma^{*}\right)$ State. Faraday Discuss. 2012, 157, 165. https://doi.org/10.1039/c2fd20076g.

(50) Roberts, G. M.; Williams, C. A.; Young, J. D.; Ullrich, S.; Paterson, M. J.; Stavros, V. G. Unraveling Ultrafast Dynamics in Photoexcited Aniline. J. Am. Chem. Soc. 2012, 134 (30), 12578-12589. https://doi.org/10.1021/ja3029729.

(51) Jhang, W. R.; Lai, H. Y.; Lin, Y.-C.; Lee, C.; Lee, S.-H.; Lee, Y.-Y.; Ni, C.-K.; Tseng, C.-M. Triplet vs $\pi \sigma^{*}$ State Mediated N-H Dissociation of Aniline. J. Chem. Phys. 2019, 151 (14), 141101. https://doi.org/10.1063/1.5121350.

(52) Bao, J. J.; Zhou, C.; Varga, Z.; Kanchanakungwankul, S.; Gagliardi, L.; Truhlar, D. G. Multi-State PairDensity Functional Theory. Faraday Discuss. 2020. https://doi.org/10.1039/D0FD00037J.

(53) Fdez. Galván, I.; Vacher, M.; Alavi, A.; Angeli, C.; Aquilante, F.; Autschbach, J.; Bao, J. J.; Bokarev, S. I.; Bogdanov, N. A.; Carlson, R. K.; Chibotaru, L. F.; Creutzberg, J.; Dattani, N.; Delcey, M. G.; Dong, S. S.; Dreuw, A.; Freitag, L.; Frutos, L. M.; Gagliardi, L.; Gendron, F.; Giussani, A.; González, L.; Grell, G.; Guo, M.; Hoyer, C. E.; Johansson, M.; Keller, S.; Knecht, S.; Kovačević, G.; Källman, E.; Li Manni, G.; Lundberg, M.; Ma, Y.; Mai, S.; Malhado, J. P.; Malmqvist, P. Å.; Marquetand, P.; Mewes, S. A.; Norell, J.; Olivucci, M.; Oppel, M.; Phung, Q. M.; Pierloot, K.; Plasser, F.; Reiher, M.; Sand, A. M.; Schapiro, I.; Sharma, P.; Stein, C. J.; Sørensen, L. K.; Truhlar, D. G.; Ugandi, M.; Ungur, L.; Valentini, A.; Vancoillie, S.; Veryazov, V.; Weser, O.; Wesołowski, T. A.; Widmark, P.-O.; Wouters, S.; Zech, A.; Zobel, J. P.; Lindh, R. OpenMolcas: From Source Code to Insight. J. Chem. Theory Comput. 2019, 15 (11), 5925-5964. https://doi.org/10.1021/acs.jctc.9b00532.

(54) Barca, G. M. J.; Bertoni, C.; Carrington, L.; Datta, D.; De Silva, N.; Deustua, J. E.; Fedorov, D. G.; Gour, J. R.; Gunina, A. O.; Guidez, E.; Harville, T.; Irle, S.; Ivanic, J.; Kowalski, K.; Leang, S. S.; Li, H.; Li, W.; Lutz, J. J.; Magoulas, I.; Mato, J.; Mironov, V.; Nakata, H.; Pham, B. Q.; Piecuch, P.; Poole, D.; Pruitt, S. R.; Rendell, A. P.; Roskop, L. B.; Ruedenberg, K.; Sattasathuchana, T.; Schmidt, M. W.; Shen, J.; Slipchenko, L.; Sosonkina, M.; Sundriyal, V.; Tiwari, A.; Galvez Vallejo, J. L.; Westheimer, B.; Włoch, M.; Xu, P.; Zahariev, F.; Gordon, M. S. Recent Developments in the General Atomic and Molecular Electronic Structure System. J. Chem. Phys. 2020, 152 (15), 154102.

https://doi.org/10.1063/5.0005188.

(55) Carlson, R. K.; Truhlar, D. G.; Gagliardi, L. Multiconfiguration Pair-Density Functional Theory: A Fully Translated Gradient Approximation and Its Performance for Transition Metal Dimers and the Spectroscopy of $\mathrm{Re}_{2} \mathrm{Cl}_{8}^{2-}$. J. Chem. Theory Comput. 2015, 11 (9), 4077-4085. https://doi.org/10.1021/acs.jctc.5b00609.

(56) Roos, B. O.; Taylor, P. R.; Sigbahn, P. E. M. A Complete Active Space SCF Method (CASSCF) Using a Density Matrix Formulated Super-Cl Approach. Chem. Phys. 1980, 48 (2), 157-173. https://doi.org/10.1016/0301-0104(80)80045-0.

(57) Perdew, J. P.; Burke, K.; Ernzerhof, M. Generalized Gradient Approximation Made Simple. Phys. Rev. Lett. 1996, 77 (18), 3865-3868. https://doi.org/10.1103/PhysRevLett.77.3865.

(58) Yu, H. S.; He, X.; Li, S. L.; Truhlar, D. G. MN15: A Kohn-Sham Global-Hybrid Exchange-Correlation Density Functional with Broad Accuracy for Multi-Reference and Single-Reference Systems and Noncovalent Interactions. Chem. Sci. 2016, 7 (8), 5032-5051. 
https://doi.org/10.1039/C6SC00705H.

(59) Weigend, F.; Ahlrichs, R. Balanced Basis Sets of Split Valence, Triple Zeta Valence and Quadruple Zeta Valence Quality for H to Rn: Design and Assessment of Accuracy. Phys. Chem. Chem. Phys. 2005, 7 (18), 3297-3305. https://doi.org/10.1039/b508541a.

(60) Fedorov, D. G.; Koseki, S.; Schmidt, M. W.; Gordon, M. S. Spin-Orbit Coupling in Molecules: Chemistry beyond the Adiabatic Approximation. Int. Rev. Phys. Chem. 2003, 22 (3), 551-592. https://doi.org/10.1080/0144235032000101743.

(61) Lorquet, J. C.; Leyh-Nihant, B. Nonadiabatic Unimolecular Reactions. 1. A Statistical Formulation for the Rate Constants. J. Phys. Chem. 1988, 92 (16), 4778-4783. https://doi.org/10.1021/j100327a043.

(62) Rice, O. K.; Ramsperger, H. C. Theories of Unimolecular Gas Reactions at Low Pressures. II. J. Am. Chem. Soc. 1928, 50 (3), 617-620. https://doi.org/10.1021/ja01390a002.

(63) Kassel, L. S. Studies in Homogeneous Gas Reactions I. J. Phys. Chem. 1928, 32 (1923), 225-242.

(64) Marcus, R. A. Unimolecular Dissociations and Free Radical Recombination Reactions. J. Chem. Phys. 1952, 20 (3), 359-364. https://doi.org/10.1063/1.1700424.

(65) Gilbert, R. G.; Smith, S. C. Theory of Unimolecular and Recombination Reactions; Blackwell Scientific Publications: Boston, 1990.

(66) Truhlar, D. G.; Kuppermann, A. A Test of Transition State Theory Against Exact Quantum Mechanical Calculations. Chem. Phys. Lett. 1971, 9 (3), 269-272. https://doi.org/10.1016/00092614(71)85049-2.

(67) Garrett, B. C.; Truhlar, D. G.; Grev, R. S.; Magnuson, A. W. Improved Treatment of Threshold Contributions in Variational Transition-State Theory. J. Phys. Chem. 1980, 84 (13), 1730-1748. https://doi.org/10.1021/j100450a013.

(68) Glowacki, D. R.; Liang, C. H.; Morley, C.; Pilling, M. J.; Robertson, S. H. MESMER: An Open-Source Master Equation Solver for Multi-Energy Well Reactions. J. Phys. Chem. A 2012, 116 (38), 95459560. https://doi.org/10.1021/jp3051033.

(69) Rosenstock, H. M.; Wallenstein, M. B.; Wahrhaftig, A. L.; Eyring, H. Absolute Rate Theory for Isolated Systems and the Mass Spectra of Polyatomic Molecules. Proc. Natl. Acad. Sci. 1952, 38 (8), 667-678. https://doi.org/10.1073/pnas.38.8.667.

(70) Magee, J. L. Theory of the Chemical Reaction Rate Constant. Proc. Natl. Acad. Sci. 1952, 38 (9), 764-770. https://doi.org/10.1073/pnas.38.9.764.

(71) Lykhin, A. O.; Kaliakin, D. S.; DePolo, G. E.; Kuzubov, A. A.; Varganov, S. A. Nonadiabatic Transition State Theory: Application to Intersystem Crossings in the Active Sites of Metal-Sulfur Proteins. Int. J. Quantum Chem. 2016, 116 (10), 750-761. https://doi.org/10.1002/qua.25124.

(72) Cui, Q.; Morokuma, K.; Bowman, J.; Klippenstein, S. The Spin-Forbidden Reaction Nonadiabatic Transition State Theory and Application. J. Chem. Phys. 1999, 110 (1999), 9469-9482. 
(73) Harvey, J. N. Spin-Forbidden Reactions: Computational Insight into Mechanisms and Kinetics. Wiley Interdiscip. Rev. Comput. Mol. Sci. 2014, 4 (1), 1-14. https://doi.org/10.1002/wcms.1154.

(74) Harvey, J. Understanding the Reactivity of Transition Metal Complexes Involving Multiple Spin States. Coord. Chem. Rev. 2003, 238-239, 347-361. https://doi.org/10.1016/S00108545(02)00283-7.

(75) Jasper, A. W. Multidimensional Effects in Nonadiabatic Statistical Theories of Spin-Forbidden Kinetics: A Case Study of ${ }^{3} \mathrm{O}+\mathrm{CO} \rightarrow \mathrm{CO}_{2}$. J. Phys. Chem. A 2015, 119 (28), 7339-7351. https://doi.org/10.1021/jp512942w.

(76) Lykhin, A. O.; Varganov, S. A. Intersystem Crossing in Tunneling Regime: $\mathrm{T}_{1} \rightarrow \mathrm{S}_{0}$ Relaxation in Thiophosgene. Phys. Chem. Chem. Phys. 2020, 22 (10), 5500-5508.

https://doi.org/10.1039/c9cp06956a.

(77) Pohlman, A. J.; Kaliakin, D. S.; Varganov, S. A.; Casey, S. M. Spin Controlled Surface Chemistry: Alkyl Desorption from Si(100)-2×1 by Nonadiabatic Hydrogen Elimination. Phys. Chem. Chem. Phys. 2020, 22 (29), 16641-16647. https://doi.org/10.1039/d0cp01913e.

(78) Nikitin, E. E. Nonadiabatic Transition Near the Turning Point in Atomic Collisions. Opt. Spectrosc. 1961, 11, 246-248.

(79) Nikitin, E. E. Theory of Nonadiabatic Transitions. Recent Development of the Landau-Zener Model. In Chemische Elementarprozesse; Hartmann, H., Ed.; Springer: Berlin, 1968; pp 43-77.

(80) Nikitin, E. E.; Umanskii, S. Y. Theory of Slow Atomic Collisions; Springer-Verlag Berlin Heidelberg: New York, 1984.

(81) Delos, J. B. On the Reactions of $\mathrm{N}_{2}$ with O. J. Chem. Phys. 1973, 59 (5), 2365. https://doi.org/10.1063/1.1680345.

(82) Garrett, B. C.; Truhlar, D. G. Semiclassical Tunneling Calculations. J. Phys. Chem. 1979, 83 (22), 2921-2926. https://doi.org/10.1021/j100485a023.

(83) Melissas, V. S.; Truhlar, D. G.; Garrett, B. C. Optimized Calculations of Reaction Paths and ReactionPath Functions for Chemical Reactions. J. Chem. Phys. 1992, 96 (8), 5758-5772. https://doi.org/10.1063/1.462674.

(84) Knee, J. L.; Johnson, P. M. Triplet State Nonradiative Lifetimes of Collision-Free Aniline and AnilineArgon Complexes above the $S_{1}$ Origin. J. Chem. Phys. 1984, 80 (1), 13-17.

https://doi.org/10.1063/1.446497.

(85) Ebata, T.; Minejima, C.; Mikami, N. A New Electronic State of Aniline Observed in the Transient IR Absorption Spectrum from $S_{1}$ in a Supersonic Jet. J. Phys. Chem. A 2002, 106 (46), 11070-11074. https://doi.org/10.1021/jp021457t.

(86) Honda, Y.; Hada, M.; Ehara, M.; Nakatsuji, H. Excited and lonized States of Aniline: Symmetry Adapted Cluster Configuration Interaction Theoretical Study. J. Chem. Phys. 2002, 117 (5), 20452052. https://doi.org/10.1063/1.1487827.

(87) Thompson, J. O. F.; Saalbach, L.; Crane, S. W.; Paterson, M. J.; Townsend, D. Ultraviolet Relaxation 
Dynamics of Aniline, N, N -Dimethylaniline and 3,5-Dimethylaniline at $250 \mathrm{~nm}$. J. Chem. Phys. 2015, 142 (11). https://doi.org/10.1063/1.4914330.

(88) Sala, M.; Kirkby, O. M.; Guérin, S.; Fielding, H. H. New Insight into the Potential Energy Landscape and Relaxation Pathways of Photoexcited Aniline from CASSCF and XMCQDPT2 Electronic Structure Calculations. Phys. Chem. Chem. Phys. 2014, 16 (7), 3122-3133. https://doi.org/10.1039/c3cp54418d.

(89) Scheps, R.; Florida, D.; Rice, S. A. Influence of Large Amplitude Vibrational Motion on the Rate of Intersystem Crossing: A Study of Single Vibronic Level Fluorescence from Aniline- $h_{7}$, Aniline N,N- $d_{2}$, Aniline- $d_{5}$, and Aniline- $d^{*}{ }^{*}$. J. Chem. Phys. 1974, 61 (5), 1730-1747.

https://doi.org/10.1063/1.1682169.

(90) McGlynn, S. P.; Azumi, T.; Kinoshita, M. Molecular Spectroscopy of the Triplet State; Prentice-Hall, Inc.: Englewood Clifs, New Jersey, 1969.

(91) Mcclure, D. S. Excited Triplet States of Some Polyatomic Molecules. I. J. Chem. Phys. 1951, 19 (6), 670-675. https://doi.org/10.1063/1.1748330.

(92) Qu, Z.; Qin, Z.; Zheng, X.; Wang, H.; Yao, G.; Zhang, X.; Cui, Z. Slow-Electron Velocity-Map Imaging Study of Aniline via Resonance-Enhanced Two-Photon Ionization Method. Spectrochim. Acta Part A Mol. Biomol. Spectrosc. 2017, 173, 432-438. https://doi.org/10.1016/j.saa.2016.09.046.

(93) Sarkar, S. K.; Kastha, G. S. Environmental Effects on the Absorption and Luminescence Properties of Aromatic Amines: Aniline. Spectrochim. Acta Part A Mol. Spectrosc. 1992, 48 (11-12), 16111624. https://doi.org/10.1016/0584-8539(92)80235-O.

(94) Gée, C.; Cuisset, A.; Divay, L.; Crépin, C. Electronic Relaxation of Aniline in Argon Matrix: A Site Selective Laser Spectroscopy. J. Chem. Phys. 2002, 116 (12), 4993-5001. https://doi.org/10.1063/1.1451055.

(95) Taleb, A. M.; Mohammed, H. H. Matrix Effects on the Absorption Spectra of Benzene Trapped in Rare Gas Matrices. Chem. Phys. 1985, 97 (2-3), 391-396. https://doi.org/10.1016/03010104(85)87047-6.

(96) Castellá-Ventura, M.; Kassab, E. Comparative Semiempirical and Ab Initio Study of the Harmonic Vibrational Frequencies of Aniline-I. The Ground State. Spectrochim. Acta Part A Mol. Spectrosc. 1994, 50 (1), 69-86. https://doi.org/10.1016/0584-8539(94)80116-9.

(97) Curchod, B. F. E.; Martínez, T. J. Ab Initio Nonadiabatic Quantum Molecular Dynamics. Chem. Rev. 2018, 118 (7), 3305-3336. https://doi.org/10.1021/acs.chemrev.7b00423.

(98) Nelson, T. R.; White, A. J.; Bjorgaard, J. A.; Sifain, A. E.; Zhang, Y.; Nebgen, B.; Fernandez-Alberti, S.; Mozyrsky, D.; Roitberg, A. E.; Tretiak, S. Non-Adiabatic Excited-State Molecular Dynamics: Theory and Applications for Modeling Photophysics in Extended Molecular Materials. Chem. Rev. 2020, 120 (4), 2215-2287. https://doi.org/10.1021/acs.chemrev.9b00447.

(99) Fedorov, D. A.; Lykhin, A. O.; Varganov, S. A. Predicting Intersystem Crossing Rates with AIMS-DFT Molecular Dynamics. J. Phys. Chem. A 2018, 122 (13), 3480-3488.

https://doi.org/10.1021/acs.jpca.8b00883. 
(100) Fernández-Ramos, A.; Ellingson, B. a.; Meana-Pañeda, R.; Marques, J. M. C.; Truhlar, D. G. Symmetry Numbers and Chemical Reaction Rates. Theor. Chem. Acc. 2007, 118 (4), 813-826. https://doi.org/10.1007/s00214-007-0328-0.

(101) Hou, X. J.; Quan, P.; Höltzl, T.; Veszprémi, T.; Nguyen, M. T. Theoretical Study of Low-Lying Triplet States of Aniline. J. Phys. Chem. A 2005, 109 (45), 10396-10402.

https://doi.org/10.1021/jp0533527.

(102) Truhlar, D. G.; Hase, W. L.; Hynes, J. T. Current Status of Transition-State Theory. J. Phys. Chem. 1983, 87 (15), 2664-2682. https://doi.org/10.1021/j100238a003.

(103) Liu, Y. P.; Lynch, G. C.; Truong, T. N.; Lu, D. hong; Truhlar, D. G.; Garrett, B. C. Molecular Modeling of the Kinetic Isotope Effect for the [1,5]-Sigmatropic Rearrangement of Cis-1,3-Pentadiene. J. Am. Chem. Soc. 1993, 115 (6), 2408-2415. https://doi.org/10.1021/ja00059a041.

(104) Blancafort, L.; Ovejas, V.; Montero, R.; Fernández-Fernández, M.; Longarte, A. Triplet Mediated C$\mathrm{N}$ Dissociation versus Internal Conversion in Electronically Excited N-Methylpyrrole. J. Phys. Chem. Lett. 2016, 7 (7), 1231-1237. https://doi.org/10.1021/acs.jpclett.6b00195.

(105) Miller, W. H.; Handy, N. C.; Adams, J. E. Reaction Path Hamiltonian for Polyatomic Molecules. J. Chem. Phys. 1980, 72 (1), 99-112. https://doi.org/10.1063/1.438959.

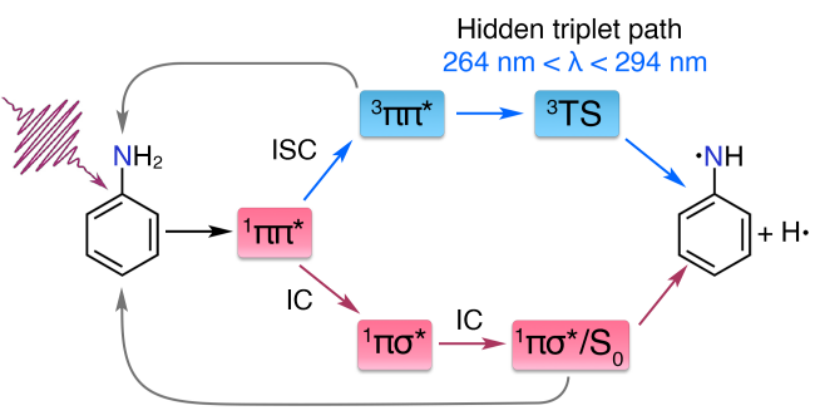

For Table of Contents Only 